\title{
JOST FUNCTIONS AND JOST SOLUTIONS FOR JACOBI MATRICES, I. A NECESSARY AND SUFFICIENT CONDITION FOR SZEGÖ ASYMPTOTICS
}

\author{
DAVID DAMANIK ${ }^{1,2}$ AND BARRY SIMON ${ }^{1,3}$
}

\begin{abstract}
We provide necessary and sufficient conditions for a Jacobi matrix to produce orthogonal polynomials with Szegö asymptotics off the real axis. A key idea is to prove the equivalence of Szegö asymptotics and of Jost asymptotics for the Jost solution. We also prove $L^{2}$ convergence of Szegö asymptotics on the spectrum.
\end{abstract}

\section{INTRODUCTION}

In 1922, Szegö [48 proved one of the most celebrated results in classical analysis: his asymptotic theorem for orthogonal polynomials. In modern language, he considered measures, $d \rho$, on $[-2,2]$ of the form

$$
d \rho(x)=f(x) d x+d \rho_{\mathrm{s}}(x)
$$

with orthonormal polynomials

$$
p_{n}(x)=\gamma_{n} x^{n}+\text { lower order }
$$

obeying $\gamma_{n}>0$ and

$$
\int p_{n}(x) p_{m}(x) d \rho(x)=\delta_{n m}
$$

What Szegö proved is that for $z \in \mathbb{D}=\{z \in \mathbb{C}|| z \mid<1\}$, one has Szegö asymptotics as $n \rightarrow \infty$

$$
z^{n} p_{n}\left(z+\frac{1}{z}\right) \rightarrow \frac{D(z)^{-1}}{\sqrt{2}}
$$

Date: March 28, 2003; February 10, 2005.

${ }^{1}$ Mathematics 253-37, California Institute of Technology, Pasadena, CA 91125. E-mail: damanik@caltech.edu; bsimon@caltech.edu.

2 Supported in part by NSF grant DMS-0227089.

${ }^{3}$ Supported in part by NSF grant DMS-0140592 and in part by Grant No. 2002068 from the United States-Israel Binational Science Foundation (BSF), Jerusalem, Israel. 
so long as the following, known as the Szegő condition, holds

$$
\int_{-2}^{2} \log f(x)\left(4-x^{2}\right)^{-1 / 2} d x>-\infty
$$

(Actually, Szegö, using the still standard convention of the orthogonal polynomial community, took $d \rho$ on $[-1,1]$ and he did not allow a singular component - that is a later refinement. Also, instead of $z \mapsto z+z^{-1}$ which maps $\mathbb{D} \rightarrow \mathbb{C} \backslash[-2,2]$, he used the inverse map and stated his results in terms of limits of

$$
\left(\frac{x}{2}+\frac{\sqrt{4-x^{2}}}{2}\right)^{n} p_{n}(x)
$$

rather than (1.4)

Szegő also found an explicit formula for $D(z)$, namely,

$$
D(z)=\exp \left[\int \frac{e^{i \theta}+z}{e^{i \theta}-z} \log (f(\cos \theta)) \frac{d \theta}{4 \pi}\right]
$$

Moreover, if (1.5) fails, so does (1.4)

From the point of view of measures, the restriction to $\operatorname{supp}(d \rho) \subset$ $[-2,2]$ is natural, but this is less so with respect to the recursion coefficients (aka Jacobi parameters) for the orthonormal polynomials, $p_{n}(x)$, defined by

$$
x p_{n}(x)=a_{n+1} p_{n+1}(x)+b_{n+1} p_{n}(x)+a_{n} p_{n-1}(x)
$$

for $\left\{a_{n}, b_{n}\right\}_{n=1}^{\infty}$. From this point of view, the natural condition is

$$
a_{n} \rightarrow 1 \quad b_{n} \rightarrow 0
$$

This is associated to, indeed implies that, $\operatorname{ess} \operatorname{supp}(d \rho)=[-2,2]$, that is, $\operatorname{supp}(d \rho)=[-2,2] \cup P$, where $P$ is a bounded set whose only possible limit points are \pm 2 . Our main goal in this paper is to answer the question of for which $\left\{a_{n}, b_{n}\right\}_{n=1}^{\infty}$ does one have Szegő asymptotics; we will find (see Theorem 5.1)

Theorem 1.1. Let $p_{n}(x)$ be orthonormal polynomials associated to Jacobi parameters $\left\{a_{n}, b_{n}\right\}_{n=1}^{\infty}$ obeying (1.9). Then $\lim z^{n} p_{n}\left(z+\frac{1}{z}\right)$ exists for all $z \in \mathbb{D}$, is nonzero for $z \in \mathbb{D} \backslash \mathbb{R}$ with convergence uniform on compacts if and only if

$$
\begin{array}{ll}
\text { ( } \alpha) & \sum_{n=1}^{\infty}\left|a_{n}-1\right|^{2}+\left|b_{n}\right|^{2}<\infty \\
(\beta) \quad & \lim _{n \rightarrow \infty} a_{n} a_{n-1} \ldots a_{1} \text { exists and is nonzero }
\end{array}
$$


( $\gamma) \quad \lim _{n \rightarrow \infty} \sum_{j=1}^{n} b_{j}$ exists

- thereby closing a chapter opened 83 years ago.

There has, of course, been prior literature on these issues, although with considerably stronger hypotheses than $(\alpha)-(\gamma)$. The initial results relating Jacobi parameters to Szegö asymptotics illustrated how strong $\operatorname{supp}(d \rho) \subset[-2,2]$ is and include

Theorem 1.2. Let $\operatorname{supp}(d \rho) \subset[-2,2]$. Then the following are equivalent:

(a) $(\beta)$ holds.

(b) $(\alpha),(\beta)$, and $(\gamma)$ hold.

(c) The Szegö condition (1.5) holds.

This theorem combines results of Shohat [37] and Nevai [30]; see also 24 and 44 . Of course, once one drops the restriction on $\operatorname{supp}(d \rho)$, the $a$ 's and $b$ 's become almost independent, and any subset of $(\alpha)-(\gamma)$ can hold.

To continue our discussion of earlier results on extending Szegö asymptotics, we need some notation. Since $P$ can only have \pm 2 as limit points,

$$
P \cap(-\infty,-2)=\left\{E_{j}^{-}\right\}_{j=1}^{N_{-}}
$$

where $N_{-}=0$ (i.e., the set is empty), $1,2, \ldots$ or $\infty$, and $E_{1}^{-}<E_{2}^{-}<$ -.. Similarly,

$$
P \cap(2, \infty)=\left\{E_{j}^{+}\right\}_{j=1}^{N_{+}}
$$

with $E_{1}^{+}>E_{2}^{+}>\cdots$. The earliest results extending Szegö asymptotics beyond $\operatorname{supp}(d \rho) \subset[-2,2]$ are due to Gonchar [16], Nevai [30], and Nikishin [31, who noted that the result still holds if $N_{+}+N_{-}<\infty$. More recently,

Theorem 1.3 (Peherstorfer-Yuditskii [32]). Suppose $a_{n} \rightarrow 1, b_{n} \rightarrow 0$, and

$$
\sum_{j, \pm}\left(\left|E_{j}^{ \pm}\right|-2\right)^{1 / 2}<\infty
$$

and that (1.5) holds. Then (1.4) holds where the function $D(z)^{-1}$ vanishes if and only if $z+z^{-1}$ is some $E_{j}^{ \pm}$.

Remark. The $D(z)^{-1}$ we use here is not the same as the $D^{-1}$ used in 32, but is a Blaschke product times their $D^{-1}$.

Related to this is 
Theorem 1.4 (Killip-Simon [24]). If

$$
\sum_{n=1}^{\infty}\left|a_{n}-1\right|+\left|b_{n}\right|<\infty
$$

then (1.13) and (1.5) hold.

From one point of view, (1.13) is quite natural. If $z_{j}^{ \pm}$is defined by

$$
z_{j}^{ \pm} \in(-1,1) \quad z_{j}^{ \pm}+\left(z_{j}^{ \pm}\right)^{-1}=E_{j}^{ \pm}
$$

then (1.13) is equivalent to

$$
\sum_{j, \pm}\left(1-\left|z_{j}^{ \pm}\right|\right)<\infty
$$

which is exactly what is needed to define a Blaschke product of zeros and obtain $D(z)^{-1}$ as a Nevanlinna function (see [32, 24, 42]). Theorems [1.3 and 1.4 are the strongest prior results on when Szegö asymptotics holds.

Both as input and motivation, the next element of background for our work concerns sum rules. Szegö proved his results for orthogonal polynomials on the real line (OPRL) by mapping the problem to one on orthogonal polynomials on the unit circle (OPUC). For OPUC, he earlier [46] proved asymptotic formulae. He began at $z=0$ where the limit formula was equivalent to his leading limit theorem for Toeplitz determinants (see [45]) and deduced the general formula from that.

Verblunsky 49] rewrote the $z=0$ limit theorem as a sum rule, namely, if

$$
d \mu(\theta)=w(\theta) \frac{d \theta}{2 \pi}+d \mu_{\mathrm{s}}
$$

is a probability measure on $\partial \mathbb{D}$ and $\alpha_{n}$ are its Verblunsky coefficients (see 41, 42] for definition), then

$$
\prod_{j=0}^{\infty}\left(1-\left|\alpha_{j}\right|^{2}\right)=\exp \left(\int \log (w(\theta)) \frac{d \theta}{2 \pi}\right)
$$

(which includes the fact that both sides are 0 simultaneously, i.e., $\left.\sum_{j=0}^{\infty}\left|\alpha_{j}\right|^{2}=\infty \Leftrightarrow \int \log (w(\theta)) \frac{d \theta}{2 \pi}=-\infty\right)$. Without knowing of Verblunsky's work, Case [3, 4], motivated by KdV sum rules, wrote some rules for Jacobi matrices with sufficiently nice $a$ 's and b's - he was not explicit about the needed conditions, but his arguments at least require

$$
\sum_{n=1}^{\infty} n\left(\left|a_{n}-1\right|+\left|b_{n}\right|\right)<\infty
$$


It was Killip-Simon 24] who realized the right combination of sum rules and proved

Theorem 1.5 (Killip-Simon [24]). Let $a_{n} \rightarrow 1$ and $b_{n} \rightarrow 0$. Then

$$
\sum_{n=1}^{\infty}\left|a_{n}-1\right|^{2}+\left|b_{n}\right|^{2}<\infty
$$

holds if and only if

$$
\sum_{j, \pm}\left(\left|E_{j}^{ \pm}\right|-2\right)^{3 / 2}<\infty
$$

and

$$
\int_{-2}^{2} \log (f(x))\left(4-x^{2}\right)^{1 / 2} d x<\infty
$$

Note that (1.22), which [24] calls the quasi-Szego" condition, is distinct from (1.5) $\left(\left(4-x^{2}\right)^{1 / 2}\right.$ rather than $\left.\left(4-x^{2}\right)^{-1 / 2}\right)$. Further developments of sum rules include [25, 26, 27, 29, 43, 44]. In particular, one has

Theorem 1.6 (Simon-Zlatoš 44). Consider the three assertions:

( $\beta) \lim _{n \rightarrow \infty} a_{n} \ldots a_{1}$ exists and is nonzero.

$(\sigma)(1.13)$ holds.

$(\tau)$ (1.5) holds.

If $(\beta)$ holds, then $(\sigma) \Leftrightarrow(\tau)$, and if $(\sigma)$ and $(\tau)$ hold, then $(\beta)$ holds.

The next element in our analysis is to link Szegő asymptotics to a different asymptotic result associated with work of Jost [20]. Jost studied certain solutions of $-u^{\prime \prime}+V u=E u$, which is the analog of

$$
a_{n} f_{n+1}+\left(b_{n}-\left(z+z^{-1}\right)\right) f_{n}+a_{n-1} f_{n-1}=0 \quad n=2,3, \ldots
$$

one of whose solutions is

$$
f_{n}(z)=p_{n-1}\left(z+\frac{1}{z}\right)
$$

As realized by Case [3, 4, 14, the analog of the Jost solution is a solution of (1.23), which is asymptotic to $z^{n}$ in the sense that

$$
z^{-n} u_{n}(z) \rightarrow 1
$$

Case showed such solutions exist if $|z|<1$ and (1.19) holds. In distinction, Szegö asymptotics says $p_{n-1}\left(z+\frac{1}{z}\right) \sim C z^{-n}$.

There may or may not be a solution of (1.23) which obeys (1.25) if one only knows $a_{n} \rightarrow 1, b_{n} \rightarrow 0$, but from either the discrete version of Weyl's analysis (see, e.g., 33, 39]) or by the Poincaré-Perron theorem (see, e.g., [42, Section 9.6]), there is a solution for $z \in \mathbb{D}$ obeying 
$f_{n} \rightarrow 0$ - indeed, obeying $f_{n+1} / f_{n} \rightarrow z$. From Weyl's point of view, this is given by the Green's function, that is, we can take it to be, for $z \in \mathbb{D} \backslash\left\{z_{j}^{ \pm}\right\}_{j=1}^{N_{ \pm}}$,

$$
w_{n}(z)=\left\langle\delta_{n},\left(z+z^{-1}-J\right) \delta_{1}\right\rangle
$$

where $J$ is the infinite Jacobi matrix

$$
J=\left(\begin{array}{cccc}
b_{1} & a_{1} & 0 & \ldots \\
a_{1} & b_{2} & a_{2} & \ldots \\
0 & a_{2} & b_{3} & \ldots \\
\ldots & \ldots & \ldots & \ldots \\
\ldots & \ldots & \ldots & \ldots
\end{array}\right)
$$

viewed as a bounded selfadjoint operator on $\ell^{2}\left(\mathbb{Z}_{+}\right)$.

We will say that Jost asymptotics occurs if for $z \in \mathbb{D} \backslash\left\{z_{j}^{ \pm}\right\}_{j=1}^{N_{ \pm}}$, $z^{-n} w_{n}(z)$ has a nonzero finite limit as $n \rightarrow \infty$. A key to our understanding of when Szegö asymptotics holds for general $a$ 's and $b$ 's (i.e., to Theorem 1.1) is the following result we prove in Section 2 .

Theorem 1.7. Fix $z_{0} \in \mathbb{D}$ so that $z_{0}+z_{0}^{-1}$ is not an eigenvalue of $J$. Then Szegö asymptotics (i.e., $z^{n} p_{n}\left(z+\frac{1}{z}\right)$ has a nonzero limit) holds at $z_{0}$ if and only if Jost asymptotics holds at $z_{0}$.

We can now turn more closely to our proof of Theorem 1.1. That Szegö asymptotics implies $(\alpha)-(\gamma)$ will be easy (and done in Section [5) once we have Theorem 1.7. Basically, $\tilde{w}_{n}(z) \equiv z^{-n} w_{n}(z)$ are analytic near $z=0$ and Jost asymptotics (uniformly on $|z|=\varepsilon$ ) implies convergence of derivatives at $z=0$. The first two Taylor terms at 0 yield $(\beta)-(\gamma)$, and as in [24], a suitable combination of the first and third Taylor coefficients is positive and yields $(\alpha)$.

The hard direction is that $(\alpha)-(\gamma)$ implies Szegö or Jost asymptotics. We will provide three distinct proofs. The first, in Section 5 , is a relative of Szegö's original proof and of the Peherstorfer-Yuditskii arguments relying on the study of analytic functions on the disk. Szegö just used (1.7) to define $D$, and Peherstorfer-Yuditskii multiplied $D^{-1}$ by a Blaschke product. We do not have either luxury here. For (1.7) to work, one needs

$$
\int \log (f(\cos \theta)) \frac{d \theta}{2 \pi}>-\infty
$$

which is equivalent to (1.5), while all we have is

$$
\int \log (f(\cos \theta)) \sin ^{2}(\theta) \frac{d \theta}{2 \pi}>-\infty
$$


which is equivalent to (1.22). Moreover, in place of (1.16), we only have

$$
\sum_{j, \pm}\left(1-\left|z_{j}\right|\right)^{3}<\infty
$$

so we cannot define a Blaschke product. The solution will be to define renormalized Blaschke products when (1.30) holds, which we do in Section 3, and a renormalized Poisson integral when (1.29) holds, which we do in Section 4. This will allow us to define a candidate for the Jost function and prove Jost asymptotics in Section 5 and so provide our first proof that $(\alpha)-(\gamma)$ imply Jost asymptotics. This proof provides bounds we will need in Section 8 to handle $L^{2}$ convergence on $\partial \mathbb{D}$.

Our second proof in Section 6 relies on an idea going back to Jost-Pais 21] that the Jost function is a Fredholm determinant. For OPRL, this is discussed in Killip-Simon 24. We will use the theory of renormalized determinants for Hilbert-Schmidt operators to construct a candidate Jost function and use it to prove Jost asymptotics.

Our final proof, in Section [7, is connected to classical results on the construction of asymptotic solutions of ODE's associated with work of Levinson 28 and Hartman-Wintner [18]; see the book of Eastham 12 . We will use results of Coffman [5] on the difference equation analogs to construct Jost solutions when $(\alpha)-(\gamma)$ hold. This construction shows that the "hard" part of Theorem 1.1] is related to known results on ODE's with $L^{2}$ perturbations. From this point of view, our contribution here is the realization that Jost solutions imply Szegö asymptotics and that the conditions are not only sufficient but necessary.

In Section 8, we discuss $L^{2}$ convergence on $\partial \mathbb{D}$, following the original scheme of Szegő [46] but with some severe technical complications because the Jost function is not Nevanlinna. This is the hardest argument in the paper. In Section 9] we provide examples for each $p<\frac{3}{2}$ of Jacobi matrices with Szegö asymptotics, but with $\sum_{j, \pm}\left(\left|E_{j}^{ \pm}\right|-2\right)^{p}=\infty$. In Section 10, we make some remarks about Schrödinger operators with $L^{2}$ potentials.

We announced our results in [7] written in September of 2003 and mentioned our $L^{2}$ results but not their proof to Serguei Denisov. In May of 2004, Denisov-Kupin 10 released a preprint discussing modified Szegö asymptotics for certain OPUC when the Szegö condition fails but a condition like (1.29) holds. Their results are quite distinct from ours although, via (1.29), there is some overlap. Many of the methods are similar - in particular, like we do in Section 4, they use renormalized Poisson representations. There is also some overlap in the $L^{2}$ control of the boundary values which we consider in Section 8. In particular, by 
using some of their ideas, it is likely we could streamline the proof of and slightly strengthen our estimate, Proposition 8.2. We have kept our original proof. We would emphasize that our work on these methods is independent and roughly simultaneous.

It is a pleasure to thank M. Moszyński and R. Romanov for useful discussions. B.S. completed this work during his stay as a Lady Davis Visiting Professor at Hebrew University, Jerusalem. He would like to thank H. Farkas and Y. Last for the hospitality of the Mathematics Institute at Hebrew University.

\section{Szegő Asymptotics And Jost Asymptotics}

As explained in the introduction, for any Jacobi matrix with $a_{n} \rightarrow 1$, $b_{n} \rightarrow 0$, and $z \in \mathbb{D}$, and not such that $z+z^{-1}$ is an eigenvalue of $J$, there are two natural solutions of

$$
a_{n} f_{n+1}+\left(b_{n}-\left(z+z^{-1}\right)\right) f_{n}+a_{n-1} f_{n-1}=0 \quad n=2,3, \ldots
$$

One is the orthogonal polynomial solution, $f_{n}=p_{n-1}\left(z+\frac{1}{z}\right)$, and the other is the Weyl solution,

$$
w_{n}(z)=\left\langle\delta_{n},\left(z+z^{-1}-J\right)^{-1} \delta_{1}\right\rangle
$$

In this section, our purpose is to show that for each such $z$, one has Jost asymptotics at that $z$, that is

$$
\tilde{w}_{n}(z) \equiv z^{-n} w_{n}(z) \rightarrow \tilde{w}_{\infty}(z)
$$

for $\tilde{w}_{\infty} \neq 0$ if and only if one has Szegö asymptotics for that $z$, that is,

$$
c_{n}(z) \equiv z^{n} p_{n}\left(z+\frac{1}{z}\right) \rightarrow c_{\infty}(z)
$$

for $c_{\infty} \neq 0$, and moreover,

$$
\left(1-z^{2}\right) c_{\infty}(z) \tilde{w}_{\infty}(z)=1
$$

(as we will see, $\tilde{w}_{\infty}(z)=1 / u(z)$, where $u$ is the Jost function, so (2.5) is usually written $\left.c_{\infty}(z)=u(z) /\left(1-z^{2}\right)\right)$.

Of course, $p_{-1}$ obeys (2.1) also at $n=1$ if we define $p_{-1} \equiv f_{0}=0$ and $a_{0}=1$. Since

$$
\left(J-z-z^{-1}\right)\left(z+z^{-1}-J\right) \delta_{1}=-\delta_{1}
$$

$w_{n}$ also obeys (2.1) if we set $a_{0}=1$ and

$$
w_{0}(z)=1
$$

The constancy of the Wronskian thus implies

$$
a_{n}\left(p_{n}\left(z+\frac{1}{z}\right) w_{n}(z)-w_{n+1}(z) p_{n-1}\left(z+\frac{1}{z}\right)\right)=1
$$


where we get 1 since

$$
a_{0}\left(p_{0} w_{0}-w_{1} p_{-1}\right)=1
$$

Using the definitions (2.3) / (2.4) of $c$ and $\tilde{w}$, (2.7) becomes

$$
a_{n}\left(c_{n}(z) \tilde{w}_{n}(z)-z^{2} \tilde{w}_{n+1}(z) c_{n-1}(z)\right)=1
$$

Thus, the following lemma is of relevance:

Lemma 2.1. Let $x_{n}, y_{n}$ be sequences of nonzero complex numbers and let $\lambda_{n}$ be nonzero positive numbers with

$$
\lambda_{n} \rightarrow 1
$$

and so, for some $z \in \mathbb{D}$,

$$
x_{n+1} y_{n}-z^{2} x_{n} y_{n+1}=\lambda_{n}
$$

Then

(i) If $y_{n} \rightarrow y_{\infty} \neq 0$, then $x_{n} \rightarrow 1 / y_{\infty}\left(1-z^{2}\right)$.

(ii) If $x_{n} \rightarrow x_{\infty} \neq 0$ and $z^{2 n} y_{n} \rightarrow 0$, then $y_{n} \rightarrow 1 / x_{\infty}\left(1-z^{2}\right)$.

Proof. (i) Rewrite (2.10) as

$$
x_{n+1}=\lambda_{n} y_{n}^{-1}+z^{2} \frac{y_{n+1}}{y_{n}} x_{n}
$$

and iterate $\ell+1$ times to get

$$
x_{n+1}=\sum_{j=0}^{\ell} \lambda_{n-j} \frac{y_{n+1}}{y_{n+1-j} y_{n-j}} z^{2 j}+z^{2 \ell+2} \frac{y_{n+1}}{y_{n-\ell}} x_{n-\ell}
$$

Set $\ell=n-1$ and see that since $\sum_{j=0}^{n-1} z^{2 j}=\left(1-z^{2 n}\right) /\left(1-z^{2}\right)$,

$$
\left|x_{n+1}-y_{\infty}^{-1}\left(1-z^{2 n}\right)\left(1-z^{2}\right)^{-1}\right| \leq \sum_{j=0}^{n-1} e_{n, j} z^{2 j}+e_{n, n} z^{2 n}
$$

where

$$
\begin{aligned}
& e_{n, j}=\lambda_{n-j} \frac{y_{n+1}}{y_{n+1-j} y_{n-j}}-\frac{1}{y_{\infty}} \quad j=0, \ldots, n-1 \\
& e_{n, n}=y_{n+1} x_{1} y_{1}^{-1}
\end{aligned}
$$

Since $y_{\ell} \rightarrow y_{\infty} \neq 0, \sup _{n, j} e_{n, j}<\infty$ and moreover, $\lim _{n \rightarrow \infty} e_{n, j}=0$ for all fixed $j$. Thus, since $e_{n, j} \rightarrow 0$ for $j$ fixed, we have for $\ell$ fixed,

$$
\begin{aligned}
\limsup _{n \rightarrow \infty}\left|\sum_{j=0}^{n} e_{n, j} z^{2 j}\right| & \leq \limsup \left|\sum_{j=\ell}^{n} e_{n, j} z^{2 j}\right| \\
& \leq|z|^{2 \ell}\left(1-|z|^{2}\right)^{-1} \sup _{n, j}\left|e_{n, j}\right|
\end{aligned}
$$


$\rightarrow 0$ as $\ell \rightarrow \infty$. Thus (2.12) implies

$$
x_{n+1} \rightarrow y_{\infty}^{-1}\left(1-z^{2}\right)^{-1}
$$

(ii) Rewrite (2.10) as

$$
y_{n}=\lambda_{n} x_{n+1}^{-1}+z^{2} x_{n} x_{n-1}^{-1} y_{n+1}
$$

and iterate upwards. Since $z^{2 n} y_{n} \rightarrow 0$, the remainder after $\ell$ iterations goes to zero as $\ell \rightarrow \infty$, so

$$
y_{n}=\sum_{j=0}^{\infty} \lambda_{n+j} z^{2 j} x_{n} x_{n+j+1}^{-1} x_{n+j}^{-1}
$$

As in the argument in (i), this implies that $y_{n} \rightarrow x_{\infty}^{-1}\left(1-z^{2}\right)^{-1}$.

Theorem 2.2 (Szegö asymptotics $=$ Jost asymptotics). Let $J$ be a Jacobi matrix with $a_{n} \rightarrow 1, b_{n} \rightarrow 0$, and let $z \in \mathbb{D}$ be such that $z+z^{-1}$ is not an eigenvalue of $J$. Then $\tilde{w}_{n}(z)$ has a nonzero limit if and only if $c_{n}(z)$ has a nonzero limit, and if either happens,

$$
\lim _{n \rightarrow \infty} c_{n}(z)=\frac{u(z)}{1-z^{2}}
$$

where

$$
u(z)^{-1} \equiv \lim _{n \rightarrow \infty} \tilde{w}_{n}(z)
$$

Proof. By (2.8), if $\lambda_{n}=a_{n}^{-1}, x_{n}=c_{n-1}(z), y_{n}=\tilde{w}_{n}(z)$, then Lemma2.1 implies this result so long as

$$
\lim _{n \rightarrow \infty} z^{2 n} \tilde{w}_{n}(z)=0
$$

But

$$
z^{2 n} \tilde{w}_{n}(z)=z^{n} w_{n}(z)
$$

goes to zero since both $w_{n} \rightarrow 0$ and $z^{n} \rightarrow 0$.

\section{Renormalized Blaschke Products}

As explained in the introduction, we need a renormalized Blaschke product that works for real zeros that only obey $\sum_{n}\left(1-\left|z_{n}\right|\right)^{3}<\infty$ rather than the usual Blaschke condition $\sum_{n}\left(1-\left|z_{n}\right|\right)<\infty$.

One can make a case that the first renormalization in science was the Weierstrass product formula - to get an analytic function vanishing at $\left\{z_{j}\right\}_{j=1}^{\infty}$ with $\left|z_{j}\right| \rightarrow \infty$, one modifies one's first guess

$$
f(z)=\prod_{j=1}^{\infty}\left(1-\frac{z}{z_{j}}\right)
$$


to

$$
f(z)=\prod_{j=1}^{\infty} W_{n_{j}}\left(\frac{z}{z_{j}}\right)
$$

where

$$
W_{n}(z)=(1-z) \exp \left(\sum_{k=1}^{n} \frac{z^{k}}{k}\right)
$$

picking the argument to be the truncation of the power series for $-\log (1-z)$. It is well known, of course, that (3.1) converges if $n_{j}$ is chosen so that $\sum\left|r / z_{j}\right|^{n_{j}+1}<\infty$ for all $r>0$. Similarly, if our only goal were to get a function with zeros in the right place, things would be easy - for one can show that if $z_{j} \in \mathbb{D},\left|z_{j}\right| \rightarrow 1$ as $j \rightarrow \infty$ and $w_{j}=z_{j} /\left|z_{j}\right|$, and if $n_{j}$ is chosen so that $\sum_{j=1}^{\infty}\left(\frac{1-\left|z_{j}\right|}{\varepsilon}\right)^{n_{j}+1}<\infty$ for all $\varepsilon>0$, then

$$
f(z)=\prod_{j=1}^{\infty} W_{n_{j}}\left(\frac{w_{j}-z_{j}}{w_{j}-z}\right)
$$

is a product converging absolutely to a nonzero function analytic in $\mathbb{D}$ with zeros at $\left\{z_{j}\right\}$.

We want our Blaschke products to have magnitude one on $\partial \mathbb{D}$ and we will want that for our renormalized Blaschke products.

For $p=0, b(z, p)=z$ : if $p \in \mathbb{D}, p \neq 0$,

$$
b(z, p)=\frac{|p|}{p} \frac{p-z}{1-\bar{p} z}
$$

so $b(0, p)=|p|>0$. The key, of course, is that $b(z, p)=0$ if and only if $z=p$ and

$$
\left|b\left(e^{i \theta}, p\right)\right|=1
$$

If $p=(1-x) \omega$ with $|\omega|=1$ and $x \in(0,1)$,

$$
\begin{aligned}
b(z,(1-x) \omega) & =\frac{1-x-z \omega^{-1}}{1-(1-x) \omega^{-1} z} \\
& =\frac{1-\frac{x}{1-\omega^{-1} z}}{1+\frac{x \omega^{-1} z}{1-\omega^{-1} z}}
\end{aligned}
$$

(3.6) shows immediately if $|z|<1$ and $\sum\left|x_{j}\right|<\infty$, then $\prod_{j=1}^{\infty} b(z,(1-$ $\left.x_{j}\right) \omega_{j}$ ) converges absolutely (and uniformly on $|z|<1-\delta$ ) since the numerators and denominators in (3.5) separately do.

(3.6) Suggests what to do if $\sum\left|x_{j}\right|^{n+1}<\infty$. Define

$$
b_{n}(z,(1-x) \omega)=\frac{W_{n}\left(\frac{x}{1-\omega^{-1} z}\right)}{W_{n}\left(\frac{-x \omega^{-1} z}{1-\omega^{-1} z}\right)}
$$


Here is the key fact:

Proposition 3.1. (a) Let $\delta>0$. Then for $|z|<1-\delta$ and $|x|<\delta / 2$,

$$
\left|b_{n}(z,(1-x) \omega)-1\right| \leq 4 \delta^{-n-1} x^{n+1}
$$

(b) For $e^{i \theta} \neq \omega$,

$$
\left|b_{n}\left(e^{i \theta},(1-x) \omega\right)\right|=1
$$

Warning. One cannot use the maximum principle and (3.9) to conclude that $\left|b_{n}(z,(1-x) \omega)\right| \leq 1$. Indeed, for $n \geq 1$,

$$
\lim _{r \uparrow 1}\left|b_{n}(r \omega,(1-x) \omega)\right|=\infty
$$

This is where $b_{n}$ 's differ from ordinary Blaschke factors. They have very singular inner factors (indeed, for $n \geq 3$, ones whose boundary values are not even signed measures).

Proof. (a) It is known, (e.g., Rudin [34, p. 301]) that

$$
|z|<1 \Rightarrow\left|W_{n}(z)-1\right| \leq|z|^{n+1}
$$

If $|x|<\delta / 2$ and $|z|<1-\delta$, then $\left|x /\left(1-\omega^{-1} z\right)\right| \leq|x / \delta|<\frac{1}{2}$ so (3.10) can be used, and if $N$ and $D$ are the numerator and denominator in (3.7), $|D|>\frac{1}{2}$. Since

$$
\left|\frac{N}{D}-1\right| \leq \frac{1}{|D|}(|N-1|+|D-1|)
$$

(3.10) implies that

$$
\left|b_{n}(z,(1-x) \omega)\right| \leq 2\left[\left|\frac{x}{\left(1-\omega^{-1} z\right)}\right|^{n+1}+\left|\frac{x \omega^{-1} z}{\left(1-\omega^{-1} z\right)}\right|^{n+1}\right]
$$

which yields (3.8).

(b) By (3.6), if $e^{i \theta} \neq \omega, b\left(e^{i \theta},(1-x) \omega\right)$ can be defined as a limit and (3.6) still holds and $b\left(e^{i \theta}, \omega\right)=1$. Thus for $x$ small, $\log b\left(e^{i \theta},(1-x) \omega\right)$ is analytic in $x$. By (3.4),

$$
\left|b\left(e^{i \theta}, p\right)\right|=\left|\frac{p-e^{i \theta}}{1-\bar{p} e^{i \theta}}\right|=\left|\frac{p-e^{i \theta}}{\bar{p}-e^{-i \theta}}\right|=1
$$

so for $x$ positive, $e^{i \theta} \neq \omega$,

$$
\operatorname{Re} \log \left(b\left(e^{i \theta},(1-x) \omega\right)\right)=0
$$

It follows that its Taylor coefficients,

$$
\log \left(b\left(e^{i \theta},(1-x) \omega\right)\right)=\sum_{n=1}^{\infty} \gamma_{n}\left(e^{i \theta}, \omega\right) x^{n}
$$


have $\gamma_{n}$ pure imaginary. Since

$$
b_{n}\left(e^{i \theta},(1-x) \omega\right) \equiv b\left(e^{i \theta},(1-x) \omega\right) \exp \left(\sum_{j=1}^{n} \gamma_{j}\left(e^{i \theta}, \theta\right) x^{j}\right)
$$

and $\gamma_{j}$ is pure imaginary, (3.9) holds.

Because we will be interested not in $b_{2}$ but something related to it by a finite correction, we need to look in detail at $\gamma_{1}$ and $\gamma_{2}$. We consider $\gamma_{j}(z, \omega)$ defined by (3.11) with $e^{i \theta} \rightarrow z$. By (3.6),

$$
\begin{aligned}
& \gamma_{1}(z, \omega)=-\left(\frac{1+\omega^{-1} z}{1-\omega^{-1} z}\right) \\
& \gamma_{2}(z, \omega)=-\frac{1}{2} \frac{\left(1-\left(\omega^{-1} z\right)^{2}\right)}{\left(1-\omega^{-1} z\right)^{2}}=-\frac{1}{2} \frac{\left(1+\omega^{-1} z\right)}{\left(1-\omega^{-1} z\right)}
\end{aligned}
$$

Remarkably, $\gamma_{1} / \gamma_{2}$ is independent of $\omega$ and $z$ ! For reasons that will be clear below, we want to consider

$$
\alpha(z)=\frac{1+z^{2}}{1-z^{2}} \quad \beta(z)=\frac{2 z}{1-z^{2}}
$$

Notice that

$$
\gamma_{1}(z, \omega= \pm 1)=2 \gamma_{2}(z, \omega= \pm 1)=-(\alpha(z) \pm \beta(z))
$$

Definition. For $p \in(-1,1), p \neq 0$, and $z \in \mathbb{D}$, we define

$$
q(z, p)=b(z, p) \exp \left(-\alpha(z) \log (|p|)-\frac{1}{2} \beta(z)\left(p-\frac{1}{p}\right)\right)
$$

Theorem 3.2. (a) For $z$ near zero and $p \neq 0, p$ real,

$$
\log q(z, p)=\log b(z, p)-\alpha(z) \log b(0, p)-\left.\frac{1}{2} \beta(z) \frac{d}{d z} \log b(z, p)\right|_{z=0}
$$

(b)

$$
q(z, p)=b_{2}(z, p) \exp \left(-\alpha(z) A(p)-\frac{1}{2} \beta(z) B(p)\right)
$$

where

$$
\begin{aligned}
& A(p)=\log |p|-(1-|p|)-\frac{(1-|p|)^{2}}{2} \\
& B(p)=-\frac{(1-|p|)^{3}}{p}
\end{aligned}
$$

(c) If $p \in(-1,1)$ with $1-|p|<\delta / 2$ and $|z|<1-\delta$, then $|q(z, p)-1| \leq\left[4 \delta^{-3}+\frac{5}{3}\left(1+4 \delta^{-3}\right) \delta^{-1}|p|^{-1} \exp \left(\frac{5}{3} \delta^{-1}|p|^{-1}(1-|p|)^{3}\right](1-|p|)^{3}\right.$ 
Proof. (a) Writing

we see

$$
b(z, p)=|p| \frac{1-\frac{z}{p}}{1-z p}
$$

$$
\log b(z, p)=\log |p|+z\left(p-\frac{1}{p}\right)+O\left(z^{2}\right)
$$

which, given (3.17), is (3.18).

(b) By (3.12) and (3.16),

$$
q(z, p)=b_{2}(z, x) \exp (C(p, z))
$$

where

$$
\begin{gathered}
C(p, z)=-\alpha(z) \log (|p|)-\frac{1}{2} \beta(z)\left(p-\frac{1}{p}\right)-\alpha(z)\left((1-|p|)+\frac{1}{2}(1-|p|)^{2}\right) \\
-\beta(z) \operatorname{sgn}(p)\left((1-|p|)+\frac{1}{2}(1-|p|)^{2}\right)
\end{gathered}
$$

Thus (3.19) follows from

$$
p-\frac{1}{p}+\operatorname{sgn}|p|\left[2(1-|p|)+(1-|p|)^{2}\right]=-\frac{(1-|p|)^{3}}{p}
$$

(3.23) follows from writing $p=\operatorname{sgn}(p)(1-x)$ and

$$
\begin{aligned}
\operatorname{sgn}(p)[(1-x) & \left.-\frac{1}{1-x}+2 x+x^{2}\right] \\
& =\operatorname{sgn}(p)\left\{(1-x)-\left[1+x+x^{2}+\frac{x^{3}}{1-x}\right]+2 x+x^{2}\right\} \\
& =-\operatorname{sgn}(p) \frac{x^{3}}{1-x}=-\frac{(1-|p|)^{3}}{p}
\end{aligned}
$$

(c) In terms of the function $C$ of (3.23),

$$
\begin{aligned}
|q(z, p)-1| & =\left|b_{2}(z, p) \exp (C(z, p))-1\right| \\
& \leq\left|b_{2}(z, p)\right||\exp (C(p, z))-1|+\left|b_{2}(z, p)-1\right|
\end{aligned}
$$

We have (3.8) to bound $\left|b_{2}(z, p)-1\right|$. Thus $\left|b_{2}\right| \leq 1+\left|b_{2}-1\right| \leq 1+4 \delta^{-3}$. Moreover,

so (3.22) follows from

$$
\left|e^{c}-1\right| \leq|c| \max \left(1,\left|e^{c}\right|\right) \leq|c| e^{|c|}
$$

$$
|C| \leq \frac{5}{3} \delta^{-1}|p|^{-1}(1-|p|)^{3}
$$

To prove (3.27), note first that $\left|1-z^{2}\right| \geq 1-|z|^{2}=(1+|z|)(1-|z|) \geq$ $\delta$. Thus

$$
|\alpha(z)| \leq \frac{2}{\delta} \quad|\beta(z)| \leq \frac{2}{\delta}
$$


Moreover, if $|p|=1-x$, then

$$
\begin{aligned}
|\log | p\left|-x-\frac{x^{2}}{2}\right| & =\left|\sum_{j=3}^{\infty} \frac{x^{j}}{j}\right| \\
& \leq \frac{1}{3} \frac{x^{3}}{1-x} \\
& =\frac{1}{3} \frac{(1-|p|)^{3}}{|p|}
\end{aligned}
$$

Thus, by (3.24) and (3.28),

$$
|C| \leq \frac{2}{\delta} \frac{(1-|p|)^{3}}{p}\left[\frac{1}{3}+\frac{1}{2}\right]=\frac{5}{3 \delta} \frac{(1-|p|)^{3}}{|p|}
$$

proving (3.27).

Because each $b_{n}(z, p)$ is unbounded on $\mathbb{D}$, the usual methods for controlling products on $\partial \mathbb{D}$ do not work; but in the case where the limit points of zeros only are a finite set, they do. Here is what we will need:

Theorem 3.3. Let $p_{n}$ be a sequence of reals in $(-1,1)$ with $\lim _{n \rightarrow \infty}\left|p_{n}\right|=1$ so that

$$
\sum_{n=1}^{\infty}\left(1-\left|p_{n}\right|\right)^{3}<\infty
$$

Let

$$
B_{\mathrm{ren}}(z)=\prod_{n=1}^{\infty} q\left(z, p_{n}\right)
$$

Then

(i) The product (3.30) converges in $\mathbb{C}_{+}=\{z \in \operatorname{Im} z>0\}$ and defines a function analytic in $\mathbb{D} \cup \mathbb{C}_{+} \cup \mathbb{C}_{-}$whose only zeros are at $\left\{p_{n}\right\}_{n=1}^{\infty}$.

$$
\left|B_{\text {ren }}\left(e^{i \theta}\right)\right|=1 \quad \theta \in(0, \pi) \cup(\pi, 2 \pi)
$$

(iii) If

$$
B_{\text {ren }}^{(N)}=\prod_{n=N+1}^{\infty} q\left(z, p_{n}\right)
$$

then for any $p<\infty$,

$$
\int\left|B_{\mathrm{ren}}^{(N)}\left(e^{i \theta}\right)-1\right|^{p} \frac{d \theta}{2 \pi} \rightarrow 0
$$


Proof. (i) If $z \in \mathbb{C}_{+}$, we have

$$
G(z) \equiv \max \left(\frac{1}{|1-z|}, \frac{1}{|1+z|}, \frac{|z|}{|1-z|}, \frac{|z|}{|1+z|}\right)<\infty
$$

Thus, if $x G(z)<1$, the arguments in $W_{n}$ in (3.7) are less than 1 and the same estimates we used to bound $|q(z, p)-1|$ still work to see

$$
|q(z, p)-1| \equiv H(z)|1-p|^{3}
$$

for suitable $H(z)$, and this shows the product converges.

(ii) Since the product converges on $\partial \mathbb{D} \backslash\{ \pm 1\}$ and $\left|q\left(e^{i \theta}, p_{n}\right)\right|=1$, (3.31) is immediate.

(iii) Since $\left|B_{\mathrm{ren}}^{(N)}\left(e^{i \theta}\right)\right|=1$, by (ii), pointwise convergence implies $L^{p}$ convergence. The estimate (3.34) implies pointwise convergence to 1 since $\sum_{n=N+1}^{\infty}\left|q\left(z, p_{n}\right)-1\right| \rightarrow 0$.

\section{Renormalized Poisson Representations}

Our goal in this section is to start out with a function, $f(z)$, on $\mathbb{D}$, which has a complex Poisson representation

$$
f(z)=\int P\left(z, e^{i \theta}\right) g\left(e^{i \theta}\right) \frac{d \theta}{2 \pi}
$$

where

$$
P\left(z, e^{i \theta}\right)=\frac{e^{i \theta}+z}{e^{i \theta}-z}
$$

and $g \in L^{1}(d \theta / 2 \pi)$, real-valued, and

$$
g\left(e^{i \theta}\right)=g\left(e^{-i \theta}\right)
$$

(so $f(z)$ is real on $\mathbb{D} \cap \mathbb{R}$ ).

We want to define

$$
h(z)=f(z)-\alpha(z) f(0)-\beta(z) f^{\prime}(0)
$$

and show it has a representation

$$
h(z)=\int Q\left(z, e^{i \theta}\right) g\left(e^{i \theta}\right) \frac{d \theta}{2 \pi}
$$

where $Q$ obeys a bound

$$
\left|Q\left(z, e^{i \theta}\right)\right| \leq C(z) \sin ^{2} \theta
$$

This will allow us to extend (4.1) to cases where one only has $\int\left|g\left(e^{i \theta}\right)\right| \sin ^{2} \theta \frac{d \theta}{2 \pi}<\infty$. In (4.4), $\alpha$ and $\beta$ are the functions in (3.15). For this section, their key property is

$$
\alpha(z) \pm \beta(z)=P\left(z, e^{i \theta}= \pm 1\right)
$$


To see why (4.6) should hold, note that, by (4.3), in (4.1) we can replace $P\left(z, e^{i \theta}\right)$ by

$$
\begin{aligned}
S\left(z, e^{i \theta}\right) & \equiv \frac{1}{2}\left[P\left(z, e^{i \theta}\right)+P\left(z, e^{-i \theta}\right)\right] \\
& =\frac{1-z^{2}}{1+z^{2}-2 z \cos \theta}
\end{aligned}
$$

Since $S\left(0, e^{i \theta}\right)=1$ and $\left.\frac{\partial}{\partial z} S\left(z, e^{i \theta}\right)\right|_{z=0}=\cos \theta$, (4.5) holds with

$$
Q\left(z, e^{i \theta}\right)=S\left(z, e^{i \theta}\right)-\alpha(z)-\beta(z) \cos \theta
$$

Because of (4.7) and $P\left(z, e^{i \theta}= \pm 1\right)=S\left(z, e^{i \theta}= \pm 1\right), Q$ vanishes at $e^{i \theta}=+1$ and at $e^{i \theta}=-1$. Since $\alpha$ is even under $\theta \rightarrow-\theta$ and $\theta \rightarrow 2 \pi-\theta$, these zeros must be quadratic, which is where (4.6) comes from.

A straightforward calculation shows that, by (4.10),

$$
\begin{aligned}
Q\left(z, e^{i \theta}\right) & =S\left(z, e^{i \theta}\right)-\frac{1+z^{2}}{1-z^{2}}-\frac{2 z \cos \theta}{1-z^{2}} \\
& =\frac{1-z^{2}}{1+z^{2}-2 z \cos \theta}-\frac{1+z^{2}+2 z \cos \theta}{1-z^{2}} \\
& =\frac{-4 z^{2} \sin ^{2} \theta}{\left(1-z^{2}\right)\left(1+z^{2}-2 z \cos \theta\right)} \\
& =\frac{-4 z^{2} \sin ^{2} \theta}{(1-z)(1+z)\left(z-e^{i \theta}\right)\left(z-e^{-i \theta}\right)}
\end{aligned}
$$

We summarize with

Theorem 4.1. Let $f$ be given by (4.1) with $g \in L^{1}(d \theta / 2 \pi)$ satisfying (4.3) and let $h(z)$ be given by (4.4). Then (4.5) holds with $Q$ given by (4.11). In particular,

$$
\left|Q\left(z, e^{i \theta}\right)\right| \leq \frac{4 \sin ^{2} \theta}{(1-|z|)^{3}}
$$

Proof. To get (4.13), note that $\left|1-z^{2}\right| \geq 1-|z|^{2}=(1-|z|)(1+|z|) \geq$ $1-|z|$ and $\left|z-e^{ \pm i \theta}\right| \geq 1-|z|$.

As a final result about renormalized Poisson representations, we note that

Theorem 4.2. Let $g \in L^{1}\left(\sin ^{2} \theta \frac{d \theta}{2 \pi}\right)$ be real-valued with $g\left(e^{i \theta}\right)=$ $g\left(e^{-i \theta}\right)$. Define

$$
f(z)=\int_{0}^{2 \pi} Q\left(z, e^{i \theta}\right) g\left(e^{i \theta}\right) \frac{d \theta}{2 \pi}
$$


Then for a.e. $\theta, \lim _{r \uparrow 1} f\left(r e^{i \theta}\right) \equiv f\left(e^{i \theta}\right)$ exists, and for a.e. $\theta$,

$$
\operatorname{Re} f\left(e^{i \theta}\right)=g\left(e^{i \theta}\right)
$$

Proof. Given $\theta_{0} \in(0, \pi)$, break the integral in (4.14) into two parts: $I_{1} \equiv\left(\theta_{0}-\delta, \theta_{0}+\delta\right) \cup\left(-\theta_{0}-\delta,-\theta_{0}+\delta\right)$ for $|\delta|<\min \left(\theta_{0}, \pi-\theta_{0}\right)$ and the complement, $I_{2}$. By (4.12), if $\theta \in I_{2}$,

$$
\mid Q\left(r e^{i \theta_{0}}, e^{i \theta} \mid \leq C \sin ^{2} \theta\right.
$$

uniformly in $r$ and $\lim _{r \uparrow 1} Q\left(r e^{i \theta_{0}}, e^{i \theta}\right)$ exists and is pure imaginary. Thus the part of the integral in (4.14) for $\theta \in I_{2}$ has a limit with real part 0 ; if $z=r e^{i \theta_{0}}, r \uparrow 1$.

On $I_{1}$, we can rewrite $Q$ as a sum of its four summands $\left(\frac{1}{2} P\left(z, e^{i \theta}\right), \frac{1}{2} P\left(z, e^{-i \theta}\right), \alpha(z)\right.$, and $\left.\beta(z) \cos \theta\right)$. Clearly, $\alpha\left(r e^{i \theta_{0}}\right)$ and $\beta\left(r e^{i \theta_{0}}\right)$ have limits which are pure imaginary. By the standard theory of Poisson kernels (Rudin [34, Duren [11]), the $P$ terms have a limit for a.e. $\theta_{0}$ whose real part is $\frac{1}{2}\left(g\left(e^{i \theta_{0}}\right)+g\left(e^{-i \theta_{0}}\right)\right)=g\left(e^{i \theta_{0}}\right)$ by the assumed symmetry of $g$.

\section{A Necessary and Sufficient Condition FOR Jost Asymptotics}

Our goal in this section is to prove that

Theorem 5.1. Let $J$ be a Jacobi matrix with $a_{n} \rightarrow 1, b_{n} \rightarrow 0$. Let $Q=\left\{z \in \mathbb{D} \mid z+z^{-1}\right.$ is an eigenvalue of $\left.J\right\}$. Then the following are equivalent:

(i) Szegö asymptotics (i.e., $z^{n} p_{n}\left(z+\frac{1}{z}\right)$ converges to a nonzero limit as $n \rightarrow \infty)$ hold for all $z \in \mathbb{D} \backslash Q$ uniformly on compact subsets of $\mathbb{D} \backslash Q$.

(ii) Szegö asymptotics hold for all $z$ with $|z|=\varepsilon$ for some $\varepsilon>0$ and uniformly in such $z$.

(iii) Jost asymptotics (i.e., $z^{-n} w_{n}(z)$ has a nonzero limit) hold for all $z \in \mathbb{D} \backslash Q$ uniformly on compact subsets of $\mathbb{D} \backslash Q$.

(iv) Jost asymptotics hold for all $z$ with $|z|=\varepsilon$ for some $\varepsilon>0$ uniformly in such $z$.

(v) The a's and b's obey three conditions:

$(\alpha)$

$$
\sum_{n=1}^{\infty}\left|a_{n}-1\right|^{2}+\sum_{n=1}^{\infty}\left|b_{n}\right|^{2}<\infty
$$

( $\beta) \lim _{n \rightarrow \infty} a_{1} \ldots a_{n}$ exists and is not zero.

$(\gamma) \lim _{n \rightarrow \infty} \sum_{j=1}^{n} b_{j}$ exists.

(vi) The spectral measure, $\mu$, on $\mathbb{R}$ and orthonormal polynomials obey the following properties: 


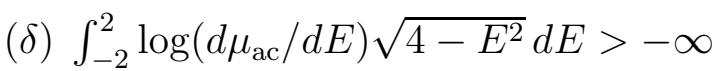

(ع) $\sum_{n}\left(\left|E_{n}^{ \pm}\right|-2\right)^{3 / 2}<\infty$

$(\kappa)$ If the orthonormal polynomials have the form

$$
p_{n}(x)=\gamma_{n}\left(x^{n}-\lambda_{n} x^{n-1}+\cdots\right)
$$

then $\lim _{n \rightarrow \infty} \gamma_{n}$ exists and is nonzero and $\lim _{n \rightarrow \infty} \lambda_{n}$ exists.

Remarks. 1. We will see shortly that $w_{n}(z)$ has an $n$ th-order zero at $z=0$, so $z^{-n} w_{n}(z)$ has a removable singularity at $z=0$ - and it is that value we intend when we say the limit exists at $z=0$.

2. We will discuss below what happens at the $z_{0}$ 's in $Q$. (Basically, $z^{n} p_{n}\left(z+\frac{1}{z}\right)$ has a zero limit there and, by shifting from Weyl to Jost solutions, we will also have control at $z_{0}$ 's in $Q$ of the other solutions.)

3 . We will see that $u(z) \equiv\left(\lim w_{\infty}(z)\right)^{-1}$ always has a factorization formula when $(\mathrm{v})$ holds. $u$ will be expressed in terms of "spectral data" and the limits in $(\beta)$ and $(\gamma)$.

Define

$$
M(z, J)=\left\langle\delta_{1},\left(z+z^{-1}-J\right)^{-1} \delta_{1}\right\rangle=w_{1}(z)
$$

Let $J^{(n)}$ be the Jacobi matrix obtained by removing the first $n$ rows and left $n$ columns of $J$. Let

$$
M_{n}(z, J)=M\left(z, J^{(n)}\right)
$$

so $M_{0}(z, J) \equiv M(z, J)$. We will often drop the $J$ if it is fixed in some discussion.

\section{Lemma 5.2.}

$$
\begin{aligned}
& M(z)=z+O\left(z^{2}\right) \\
& M_{n}(z)=\frac{w_{n+1}(z)}{a_{n} w_{n}(z)} \\
& w_{n}(z)=M(z)\left(a_{1} M_{1}(z)\right) \ldots\left(a_{n-1} M_{n-1}(z)\right) \\
& w_{n}(z)=\left(a_{1} \ldots a_{n}\right) z^{n}+O\left(z^{n+1}\right) \\
& M_{n}(z)=\left(z+z^{-1}-b_{n+1}-a_{n+1}^{2} M_{n+1}(z)\right)^{-1} \\
& \log \left(\frac{M_{n}(z)}{z}\right)=b_{n+1} z+\left(\frac{1}{2} b_{n+1}^{2}+a_{n+1}^{2}-1\right) z^{2}+O\left(z^{3}\right)
\end{aligned}
$$

Remark. Some of these equalities are intended in the sense of the field of meromorphic functions. For example, if $\ell<n$ and $w_{\ell}\left(z_{0}\right)=0$, then $M_{\ell}(z)$ has a pole at $z_{0}$ and $M_{\ell-1}(z)$ a zero there and they are intended to cancel in (5.7). Alternatively, these formulae hold initially away 
from $\left\{z \in \mathbb{D} \mid z+z^{-1} \in \sigma\left(J^{(\ell)}\right)\right.$ for some $\left.\ell=0,1, \ldots\right\}$ and then they have removable singularities in some cases.

Proof. (i) $M(z) / z=\left\langle\delta_{1},\left(1+z^{2}-z J\right)^{-1} \delta_{1}\right\rangle=1+O(z)$ as $z \rightarrow 0$.

(ii) As noted in Section 2, $w_{n}(z)$ is normalized by (2.6), that is, by

$$
a_{1} w_{2}(z)+\left(b_{1}-z-z^{-1}\right) w_{1}(z)=-1
$$

and, of course, $M(z)=w_{1}(z)$. (5.6) thus follows from

$$
a_{n+1}\left(\frac{w_{n+2}}{a_{n} w_{n}}\right)+\left(b_{n+1}-z-z^{-1}\right)\left(\frac{w_{n+1}}{a_{n} w_{n}}\right)=-1
$$

since $w_{n+j} / a_{n} w_{n}$ solves the difference equation for $J^{(n)}$.

(iii) follows from (5.6) and $w_{1}=M$.

(iv) is immediate from (5.5) for $M_{n}(z)$ and (5.7).

(v) follows from (5.6) and the difference equation for $w$.

(vi) From (5.9) for $n=0$ and (5.5) for $M_{1}$,

$$
\frac{M(z)}{z}=\left(1-b_{1} z-a_{1} z^{2}+z^{2}+O\left(z^{3}\right)\right)^{-1}
$$

so

$$
\begin{aligned}
\log \left(\frac{M(z)}{z}\right) & =-\log \left(1-b_{1} z-a_{1}^{2} z^{2}+z^{2}+O\left(z^{3}\right)\right) \\
& =\left(b_{1} z+\frac{1}{2} b_{1} z^{2}\right)+\left(a_{1}^{2}-1\right) z^{2}+O\left(z^{3}\right)
\end{aligned}
$$

Reduction of Theorem 5.1 to (v) $\Rightarrow$ (iii). By Theorem 2.2. (i) $\Leftrightarrow$ (iii) and (ii) $\Leftrightarrow$ (iv). (iii) $\Rightarrow$ (iv) is trivial. Thus we need to prove (iv) $\Rightarrow$ $(\mathrm{v})$ and $(\mathrm{v}) \Leftrightarrow(\mathrm{vi})$ to reduce the proof to $(\mathrm{v}) \Rightarrow$ (iii).

The equivalence of (v) and (vi) is easy, given the result of KillipSimon [24. They prove that $(\alpha) \Leftrightarrow(\delta),(\varepsilon)$. The equivalence of $(\kappa)$ and $(\beta),(\gamma)$ is immediate since the recursion relations for $p$ imply that

$$
\begin{aligned}
a_{n+1} \gamma_{n+1} & =\gamma_{n} \\
-\lambda_{n+1} & =-\lambda_{n}-b_{n+1}
\end{aligned}
$$

SO

$$
\gamma_{n}=\left(a_{1} \ldots a_{n}\right)^{-1} \quad \lambda_{n}=\sum_{j=1}^{n} b_{j}
$$

To study (iv) $\Rightarrow(\mathrm{v})$, define

$$
\tilde{w}_{n}(z)=z^{-n} w_{n}(z) \quad \tilde{M}_{n}(z)=z^{-1} M_{n}(z)
$$


so, by (5.7),

$$
\log \tilde{w}_{n}(z)=\sum_{j=1}^{n-1} \log \left(a_{j}\right)+\sum_{j=1}^{n-1} \log \tilde{M}_{j-1}(z)
$$

Convergence of $\tilde{w}_{n}(z)$ uniformly on the circle and analyticity of $\tilde{w}_{n}(z)$ implies the derivatives of $\tilde{w}_{n}(z)$ at $z=0$ all converge. By (5.10) and (5.13), the terms of order $1, z, z^{2}$ yield

$$
\begin{gathered}
\lim _{n \rightarrow \infty} \sum_{j=1}^{n-1} \log \left(a_{j}\right)=\nu_{1} \\
\lim _{n \rightarrow \infty} \sum_{j=1}^{n-1} b_{j}=\nu_{2} \\
\lim _{n \rightarrow \infty} \sum_{j=1}^{n-1} a_{j}^{2}-1+\frac{1}{2} b_{j}^{2}=\nu_{3}
\end{gathered}
$$

all exist. Following Killip-Simon [24, we look at (5.16) $-2 \times(\underline{5.14})$ to see

$$
\lim _{n \rightarrow \infty} \sum_{j=1}^{n-1} G\left(a_{j}\right)+\frac{1}{2} b_{j}^{2}=\nu_{3}-2 \nu_{1}
$$

where

$$
G(a)=a^{2}-1-2 \log (a)
$$

Since $G(a)>0$ for $a \in(0, \infty)$, the summand in (5.17) is nonnegative, so (5.17) implies absolute convergence. Since $G(a) \geq(a-1)^{2}$ (for $G(1)=G^{\prime}(1)=0$ and $G^{\prime \prime}(a) \geq 2$ ), (5.17) implies

$$
\sum_{j=1}^{\infty}\left(a_{j}-1\right)^{2}+\frac{1}{2} b_{j}^{2}<\infty
$$

which is $(\alpha) .(\beta)$ is the exponential of (5.14) and $(\gamma)$ is (5.15).

We now turn towards proving Jost asymptotics when $(\alpha),(\beta),(\gamma)$ hold. We will give three proofs: one in this section using canonical factorization of $M$-functions, one in Section 6 using renormalized determinants, and one in Section [ using Levinson-type asymptotic analysis of difference equations.

Our starting point for the proof in this section will be the "nonlocal" step-by-step sum rule of Simon [43]:

Theorem 5.3. For any Jacobi matrix with $a_{n} \rightarrow 1, b_{n} \rightarrow 0$,

$$
\left\{\theta \mid \operatorname{Im} M_{n}(\theta) \neq 0\right\}=\left\{\theta \mid \operatorname{Im} M_{n+1}(\theta) \neq 0\right\}
$$


(modulo sets of $d \theta / 2 \pi$-measure zero). For any $p<\infty$,

$$
\log \left(\frac{\operatorname{Im} M_{n}(\theta)}{\operatorname{Im} M_{n-1}(\theta)}\right) \in L^{p}\left(\partial \mathbb{D}, \frac{\partial \theta}{2 \pi}\right)
$$

and

$$
a_{n+1} M_{n}(z)=z B_{n}^{+} B_{n}^{-}(z) \exp \left(\frac{1}{4 \pi} \int \log \left(\frac{\operatorname{Im} M_{n}(z)}{\operatorname{Im} M_{n+1}(z)}\right)\left(\frac{e^{i \theta}+z}{e^{i \theta}-z}\right) d \theta\right)
$$

Here $B_{n}^{ \pm}$are alternating Blaschke products $\left(B^{+}\right.$for $0<p_{1,+}^{(n)}<z_{1,+}^{(n)}<$ $\cdots<p_{\ell,+}^{(n)}<z_{\ell,+}^{(n)}$ and $B^{-}$for $\left.0>p_{1,-}^{(n)}>z_{1,-}^{(n)}>\cdots\right)$ with $p_{j, \pm}^{(n)}+p_{j, \pm}^{(n)-1}$ the eigenvalues of $J^{(n)}$ and $z_{j, \pm}^{(n)}+z_{j, \pm}^{(n)-1}$ the eigenvalues of $J^{(n+1)}$.

Remarks. 1. (5.20) is a special case of a general factorization theorem for meromorphic Herglotz functions, $f$, of $\mathbb{D}$. The general theorem has $\frac{1}{2 \pi} \log \left(\left|f\left(e^{i \theta}\right)\right|\right)$. (5.20) then follows from $\left|a_{n+1} M_{n}\right|^{2}=\operatorname{Im} M_{n} / \operatorname{Im} M_{n+1}$, which is a consequence of (5.9)).

2. In our applications, the set in (5.18) is all of $\partial \mathbb{D}$. When this is false, $\operatorname{Im} M_{n} / \operatorname{Im} M_{n+1}$ in (5.19) and (5.20) have to be suitably defined on the complement of the set in (5.18); see [43] for details.

We define

$$
\begin{aligned}
L_{n}(z) & =\log \left(\frac{a_{n+1} M_{n}(z)}{z}\right) \\
N_{n}(z) & =L_{n}(z)-\alpha(z) L_{n}(0)-\frac{1}{2} \beta(z) L_{n}^{\prime}(0)
\end{aligned}
$$

where $\alpha, \beta$ are given by (3.15). If $p_{1, \pm}^{(n)}$ are the poles of $M_{n}$ closest to $z=0$, we define $L_{n}(z)$ unambiguously on $\mathbb{D} \backslash\left[p_{1,+}^{(n)}, 1\right) \cup\left(-1, p_{1,-}^{(n)}\right]$ by requiring $L_{n}(z)$ analytic and $L_{n}(0)$ real. Since $p_{1, \pm}^{(n)} \rightarrow \pm 1$ as $n \rightarrow \infty$, the result below exponentiated holds on $\mathbb{D} \backslash\left\{p_{j, \pm}^{(0)} \mid j=1,2, \ldots\right\}$.

Lemma 5.4. Suppose that $(\alpha)$, that is, (5.1) holds. Then for all $z \in$ $\mathbb{D} \backslash\left[p_{1,+}^{(0)}, 1\right) \cup\left(-1, p_{1,+}^{(0)}\right]=S$,

$$
\lim _{N \rightarrow \infty} \sum_{n=0}^{N} N_{n}(z)
$$

exists and the convergence is uniform on compact subsets of $S$.

Proof. By (5.20),

$$
L_{n}(z)=\log B_{n}^{+}(z)+\log B_{n}^{-}(z)+\frac{1}{4 \pi} \int\left(\frac{e^{i \theta}+z}{e^{i \theta}-z}\right) \log \left(\frac{\operatorname{Im} M_{n}}{\operatorname{Im} M_{n+1}}\right) d \theta
$$


Using (3.18) and (4.4),

$$
\begin{array}{r}
N_{n}(z)=\sum_{j, \pm}\left[-\log q\left(z, p_{j, \pm}^{(n)}\right)+\log q\left(z, z_{j, \pm}^{(n)}\right)\right] \\
+\frac{1}{4 \pi} \int Q\left(z, e^{i \theta}\right) \log \left(\frac{\operatorname{Im} M_{n}}{\operatorname{Im}_{n+1}}\right) d \theta
\end{array}
$$

Since $z_{j, \pm}^{(n)}=p_{j, \pm}^{(n+1)}$, this implies

$$
\begin{aligned}
\sum_{n=0}^{N} N_{n}(z)=\sum_{j, \pm} & {\left[-\log q\left(z, p_{j, \pm}^{(0)}\right)+\log q\left(z, p_{j, \pm}^{(N+1)}\right)\right] } \\
+ & \frac{1}{4 \pi} \int Q\left(z, e^{i \theta}\right) \log \left(\frac{\operatorname{Im} M}{\operatorname{Im} M_{N+1}}\right) d \theta
\end{aligned}
$$

The Killip-Simon 24] $P_{2}$ sum rule implies

(a) $\int \sin ^{2} \theta\left|\log \left(\frac{\operatorname{Im} M}{\sin \theta}\right)\right| \frac{d \theta}{2 \pi}<\infty$

(b) $\sum\left(1-\left|p_{j, \pm}^{(0)}\right|\right)^{3}<\infty$

(c) $\lim _{N \rightarrow \infty} \int \sin ^{2} \theta\left|\log \left(\frac{\operatorname{Im} M_{N+1}}{\sin \theta}\right)\right| \frac{d \theta}{2 \pi}=0$

(d) $\lim _{N \rightarrow \infty} \sum\left(1-\left|p_{j, \pm}^{(N+1)}\right|\right)^{3}=0$

(a) and (b) and the estimates (3.22) and (4.13) allow us to write (5.25) as a difference of $M^{(0)} / p^{(0)}$ terms and $M^{(N+1)} / p^{(N+1)}$ terms and then $(\mathrm{c}),(\mathrm{d})$ show that the error terms go to zero. The result is that $\lim \sum_{n=0}^{N} N_{n}(z)$ exists and

$$
\begin{aligned}
\lim _{n \rightarrow \infty} \exp \left(\sum_{n=0}^{N} N_{n}(z)\right) \\
\quad=\prod_{j= \pm 1} q\left(z, p_{j, \pm}^{(0)}\right)^{-1} \exp \left(\frac{1}{4 \pi} \int Q\left(z, e^{i \theta}\right) \log \left(\frac{\operatorname{Im} M}{\sin \theta}\right) d \theta\right)
\end{aligned}
$$

The proof shows the convergence is uniform.

Proof of Theorem 5.1(v) $\Rightarrow$ (iii). By (5.7), with $\tilde{w}_{n}(z)=z^{-n} w_{n}(z)$,

$$
a_{n} \tilde{w}_{n}(z)=\exp \left(\sum_{j=0}^{n-1} L_{j}(z)\right)
$$

Since $a_{n} \rightarrow 1, \tilde{w}_{n}(z)$ has a nonzero limit (i.e., Jost asymptotics hold) if and only if

$$
\lim _{N \rightarrow \infty} \sum_{j=0}^{N} L_{j}(z)
$$


exists and the convergence of $\tilde{w}_{n}$ is uniform if and only if the convergence of the sum is. Since

$$
L_{j}(0)=\log \left(a_{j+1}\right)
$$

by (5.5) and

$$
L_{j}^{\prime}(0)=b_{j+1}
$$

by (5.10), we have that

$$
L_{j}(z)=N_{j}(z)+\alpha(z) \log \left(a_{j+1}\right)+\frac{1}{2} \beta(z) b_{j+1}
$$

By Lemma 5.4 $\sum_{n=0}^{N} N_{n}(z)$ converges uniformly if $(\alpha)$ holds, $(\beta)$ and $(\gamma)$ say that $\sum_{n=0}^{N} \log \left(a_{n+1}\right)$ and $\sum_{n=0}^{N} b_{j+1}$ converge so $\sum_{n=0}^{N} L_{n}(z)$ converges uniformly.

If $\tilde{w}_{n}(z)$ has a nonzero limit, we define the Jost function by

$$
u(z)=\lim _{n \rightarrow \infty} \tilde{w}_{n}(z)^{-1}
$$

This agrees with the usual definition if $\sum_{n=1}^{\infty}\left|a_{n}-1\right|+\left|b_{n}\right|<\infty$. Thus

$$
u(z)=\exp \left(-\sum_{n=0}^{\infty} L_{n}(z)\right)
$$

and we have proven (by (5.26) ) that

Theorem 5.5. If $(\alpha),(\beta),(\gamma)$ hold, then

$$
\begin{aligned}
u(z)= & \left(\prod_{j=1}^{\infty} a_{j}\right)^{-\alpha(z)} e^{-\frac{1}{2} \beta(z) \sum_{j=1}^{\infty} b_{j}} \\
& \prod_{j=1, \pm}^{\infty} q\left(z, p_{j, \pm}^{(0)}\right) \exp \left(-\frac{1}{4 \pi} \int Q\left(z, e^{i \theta}\right) \log \left(\frac{\operatorname{Im} M}{\sin \theta}\right) d \theta\right)
\end{aligned}
$$

In the above, $\prod_{j=1}^{\infty} a_{j}$ and $\sum_{j=1}^{\infty} b_{j}$ refer to the conditional limits.

The integral representation (5.31) implies

Theorem 5.6. Let $(\alpha),(\beta),(\gamma)$ hold and let

$$
u(z)=\left(\lim _{n \rightarrow \infty} z^{-n} w_{n}(n)\right)^{-1}
$$

Then

(i) After removing the removable singularities at $\left\{p_{j}^{(0)}\right\}, u(z)$ is analytic in $\mathbb{D}$ and $u\left(z_{0}\right)=0\left(z_{0} \in \mathbb{D}\right)$ if and only if $z_{0} \in\left\{p_{j}^{(0)}\right\}$, that is, if and only if $z_{0}+z_{0}^{-1}$ is an eigenvalue of $J$. 
(ii) For a.e. $\theta, \lim _{r \uparrow 1} u\left(r e^{i \theta}\right) \equiv u\left(e^{i \theta}\right)$ exists and

$$
\operatorname{Im} M\left(e^{i \theta}\right)\left|u\left(r e^{i \theta}\right)\right|^{2}=\sin \theta
$$

for a.e. $\theta$.

Proof. (i) is immediate from (5.31) and (ii) follows from the fact that $\alpha, \beta$ have purely imaginary values on $\partial \mathbb{D} \backslash\{ \pm 1\}$ from (3.31) and Theorem 4.2 .

Remark. However, unlike the case where $\sum_{n=1}^{\infty}\left|a_{n}-1\right|+\left|b_{n}\right|<\infty, u$ may not be Nevanlinna. Indeed, if $\sum_{n=1}^{\infty}\left(\left|E_{n}^{ \pm}\right|-2\right)^{1 / 2}=\infty, u$ cannot be Nevanlinna. This is the subject of Section 8

\section{Renormalized Determinants}

The idea that Jost functions are given by Fredholm determinants goes back to Jost-Pais [21, and for Jacobi matrices was made explicit by Killip-Simon [24]. They define the perturbation determinant by

$$
L(z, J)=\operatorname{det}\left(\mathbf{1}+\delta J\left(J_{0}-\left(z+z^{-1}\right)\right)^{-1}\right)
$$

where

$$
\delta J=J-J_{0}
$$

and $J_{0}$ is the Jacobi matrix associated to $a_{n} \equiv 1, b_{n} \equiv 0$. This definition is used when $z \in \mathbb{D}$ and

$$
\sum_{n=1}^{\infty}\left|a_{n}-1\right|+\left|b_{n}\right|<\infty
$$

In this case, $\delta J$ is trace class and the det in (6.1) is the standard trace class determinant (see Simon [38 and Goh'berg-Krein [15]).

What Killip-Simon 24] prove in their Theorem 2.16 is

Theorem 6.1. For $J-J_{0}$ trace class, $z \in \mathbb{D}$, and $z+z^{-1} \notin \sigma(J)$, we have that the function $M$ given by (5.4) obeys

$$
M(z, J)=\frac{z L\left(z, J^{(1)}\right)}{L(z, J)}
$$

and with $w_{n}(z)$, the Weyl solution,

$$
z^{-n} w_{n}(z) \rightarrow \frac{\left[\prod_{j=1}^{\infty} a_{j}\right]}{L(z, j)}
$$

Remarks. 1. (6.4) implies (6.5) using (5.7) and $L\left(z, J^{(n)}\right) \rightarrow 1$ since $\left\|J^{(n)}-J_{0}\right\|_{1} \rightarrow 0$. 
2. (6.5), of course, says that the Jost function is given by

$$
u(z ; J)=\left[\prod_{j=1}^{\infty} a_{j}\right]^{-1} L(z ; J)
$$

3. Formula (6.4) is an expression of Cramer's rule since, very formally, Cramer's rule says

$$
M(z, J)=\frac{\operatorname{det}\left(z+z^{-1}-J^{(1)}\right)}{\operatorname{det}\left(z+z^{-1}-J\right)}
$$

and

$$
z=M\left(z, J^{(0)}\right)=\frac{\operatorname{det}\left(z+z^{-1}-J_{0}^{(1)}\right)}{\operatorname{det}\left(z+z^{-1}-J_{0}\right)}
$$

Moreover,

$$
L(z, J)=\frac{\operatorname{det}\left(J-\left(z+z^{-1}\right)\right)}{\operatorname{det}\left(J_{0}-\left(z+z^{-1}\right)\right)}
$$

(6.7) -6.9) manipulate to (6.4). Of course, the det's in (6.7)-(6.9) are all infinite, but one way to prove (6.4) is to prove (6.7)-(6.9) for cutoff finite matrices and take limits.

When (5.1) holds, $J-J_{0}$ may not any longer be trace class, but it is Hilbert-Schmidt, which suggests that we use the renormalized determinant for such operators. Such determinants go back to Carleman [2]. They are discussed in [38, 15. Our approach, due to Seiler 35, 36] and used in [38, relies on the fact that if $A$ is Hilbert-Schmidt, then

$$
B=(1+A) e^{-A}-\mathbf{1}
$$

is trace class, so we can define

$$
\operatorname{det}_{2}(\mathbf{1}+A) \equiv \operatorname{det}(\mathbf{1}+B)=\operatorname{det}\left((\mathbf{1}+A) e^{-A}\right)
$$

It obeys (see [38, Chapter 9])

$$
\begin{gathered}
A \in \mathcal{I}_{1} \rightarrow \operatorname{det}(\mathbf{1}+A)=\operatorname{det}_{2}(\mathbf{1}+A) e^{\operatorname{Tr}(A)} \\
\left|\operatorname{det}_{2}(\mathbf{1}+A)-\operatorname{det}_{2}(\mathbf{1}+C)\right| \leq\|A-C\|_{2} \exp \left(\Gamma_{2}\left(\|A\|_{2}+\|C\|_{2}+1\right)\right)
\end{gathered}
$$

for a suitable constant, $\Gamma_{2}$.

We note (see [41, eqn. (1.2.24)]) that

$$
\left[\left(J_{0}-\left(z+z^{-1}\right)\right)^{-1}\right]_{n m}=-\left(z^{-1}-z\right)^{-1}\left[z^{|m-n|}-z^{m+n}\right]
$$

Thus

$$
\left[\delta J\left(J_{0}-\left(z+z^{-1}\right)\right)^{-1}\right]_{n n}=-\left(z^{-1}-z\right)^{-1}\left\{\left(a_{n-1}-1\right)\left(z-z^{2 n-1}\right)+b_{n}\left(1-z^{2 n}\right)+\left(a_{n}-1\right)\left(z-z^{2 n+1}\right)\right\}
$$

which implies: 
Lemma 6.2. If $\delta J$ is trace class and $z \in \mathbb{D}$, then

$\operatorname{Tr}\left(\delta J\left(J_{0}-\left(z+z^{-1}\right)\right)^{-1}\right)=-\left(z^{-1}-z\right)^{-1}\left\{\sum_{n=1}^{\infty} b_{n}\left(1-z^{2 n}\right)+2 \sum_{n=1}^{\infty}\left(a_{n}-1\right)\left(z-z^{2 n+1}\right)\right\}$

It also explains the relevance of

Proposition 6.3. Suppose $a_{n}, b_{n}$ obey $(\alpha)-(\gamma)$ of Theorem [5.1, Then $T(z ; J)=\lim _{N \rightarrow \infty}\left[-\left(z^{-1}-z\right)^{-1} \sum_{n=1}^{N} b_{n}\left(1-z^{2 n}\right)+2 \sum_{n=1}^{N}\left(a_{n}-1\right)\left(z-z^{2 n+1}\right)\right]$

exists for all $z \in \mathbb{D}$ and the convergence is uniform for compact subsets of $\mathbb{D}$.

Proof. $z^{2 \cdot} \in \ell^{2}$ for $z \in \mathbb{D}$ uniformly on compact subsets, so $(\alpha)$ implies

$$
\sum_{n=1}^{\infty} b_{n} z^{2 n} \quad \text { and } \quad \sum_{n=1}^{\infty}\left(a_{n}-1\right) z^{2 n+1}
$$

converge absolutely to an analytic limit.

$(\beta)$ plus $\sum_{n=1}^{\infty}\left|a_{n}-1\right|^{2}<\infty$ implies $\lim _{N \rightarrow \infty} \sum_{n=1}^{N}\left(a_{n}-1\right)$ exists, and this plus $(\gamma)$ implies the existence of the remaining terms.

Definition. If $(\alpha)-(\gamma)$ of Theorem 5.1 hold, we define

$$
L_{\text {ren }}(z, J)=\operatorname{det}_{2}\left(1+\delta J\left(J_{0}-\left(z+z^{-1}\right)\right)^{-1}\right) e^{T(z ; J)}
$$

Proposition 6.4. Let $z \in \mathbb{D}$. $L_{\mathrm{ren}}(z, J)=0$ if and only if $z+z^{-1} \in$ $\sigma(J)$.

Proof. $\operatorname{det}_{2}(1+A)=0$ if and only if $1+A$ is not invertible (see 38, Chapter 9]). Since $1+\delta J\left(J_{0}-\left(z+z^{-1}\right)\right)^{-1}=\left(J-\left(z+z^{-1}\right)\right)\left(J_{0}-(z+\right.$ $\left.z^{-1}\right)$ ), this happens if and only if $z+z^{-1} \in \sigma(J)$.

By (6.1), 6.11), and (6.15), we have that

Proposition 6.5. If $\sum_{n=1}^{\infty}\left|a_{n}-1\right|+\left|b_{n}\right|<\infty$, then

$$
L_{\text {ren }}(z, J)=L(z, T)
$$

Theorem 6.6. If $(\alpha)-(\gamma)$ of Theorem 5.1 hold, then for all $z \in \mathbb{D}$ so $z+z^{-1} \notin \sigma(J)$,

and

$$
M(z, J)=\frac{z L_{\mathrm{ren}}\left(z ; J^{(1)}\right)}{L_{\mathrm{ren}}(z ; J)}
$$

$$
z^{-n} w_{n}(z)=\left(\prod_{j=1}^{n-1} a_{j}\right) \frac{L_{\mathrm{ren}}\left(z ; J^{(n)}\right)}{L_{\mathrm{ren}}(z ; J)}
$$


Proof. (6.20) is implied by (6.19) and (5.7), so we need only prove (6.19). Define $J_{n}$ to be the Jacobi matrix with

$$
\begin{aligned}
& a_{j}^{(n)}= \begin{cases}a_{j} & j \leq n-1 \\
1 & j \geq n\end{cases} \\
& b_{j}^{(n)}= \begin{cases}b_{j} & j \leq n \\
0 & j \geq n+1\end{cases}
\end{aligned}
$$

Then, by $(\alpha)$,

$$
\left\|J_{n}-J\right\|_{2} \rightarrow 0
$$

so $\left\|\delta J_{n}-\delta J\right\|_{2} \rightarrow 0$, so by (6.12),

$$
\operatorname{det}_{2}\left(1+\delta J_{n}\left(J_{0}-\left(z+z^{-1}\right)\right)^{-1}\right) \rightarrow \operatorname{det}_{2}\left(1+\delta J\left(J_{0}-\left(z+z^{-1}\right)\right)^{-1}\right)
$$

It is easy to see that $T\left(z, J_{n}\right) \rightarrow T(z, J)$. Thus, using (6.18), uniformly on compacts of $\mathbb{D}$,

$$
\lim _{n \rightarrow \infty} L\left(z, J_{n}\right)=\lim _{n \rightarrow \infty} L_{\mathrm{ren}}\left(z, J_{n}\right)=L_{\mathrm{ren}}(z, J)
$$

The same is true for $J_{n}^{(1)}$ and $J^{(1)}$. Therefore, since $u(z, J) \neq 0$, 6.4 implies (6.19).

We therefore have the second proof of the hard part of Theorem 5.1 . Theorem 6.7. Let $(\alpha)-(\gamma)$ of Theorem 5.1 hold. Then uniformly on compact subsets of $\mathbb{D} \backslash\left\{z \mid z+z^{-1} \in \sigma(J)\right\}$,

$$
z^{-n} w_{n}(z) \rightarrow u(z)^{-1}
$$

where

$$
u(z)=\left(\lim _{n \rightarrow \infty} \prod_{j=1}^{n} a_{j}\right)^{-1} L_{\mathrm{ren}}(z, J)
$$

Proof. By (6.20), this is equivalent to

$$
\lim _{n \rightarrow \infty} L_{\mathrm{ren}}\left(z, J^{(n)}\right)=1
$$

uniformly on compacts. It is easy to see that $\lim _{n \rightarrow \infty} T\left(z, J^{(n)}\right)=0$. Since $\left\|J^{(n)}-J_{0}\right\|_{2} \rightarrow 0$

$$
\operatorname{det}_{2}\left(1+\delta J_{n}\left(J_{0}-\left(z+z^{-1}\right)\right)^{-1}\right) \rightarrow 1
$$

This proves (6.28). 


\section{Geronimo-Case Equations}

Given a set $\left\{a_{n}, b_{n}\right\}_{n=1}^{\infty}$ of real Jacobi parameters, the Geronimo-Case polynomials $c_{n}(z), g_{n}(z)$ are defined by the recursion relations:

$$
\begin{aligned}
& c_{n+1}(z)=a_{n+1}^{-1}\left[\left(z^{2}-b_{n+1} z\right) c_{n}(z)+g_{n}(z)\right] \\
& g_{n+1}(z)=a_{n+1}^{-1}\left[\left(\left(1-a_{n+1}^{2}\right) z^{2}-b_{n+1} z\right) c_{n}(z)+g_{n}(z)\right]
\end{aligned}
$$

with initial conditions

$$
c_{0}(z)=g_{0}(z)=1
$$

They were introduced in a slightly different form by Geronimo-Case 14 who, under a condition that $\sum n\left[\left|a_{n}-1\right|+\left|b_{n}\right|\right]<\infty$, proved that for $z \in \overline{\mathbb{D}}, \lim _{n \rightarrow \infty} g_{n}(z)$ exists and defined it to be the Jost function. In Paper II of our current series [8, we will reexamine these equations to prove convergence in $\mathbb{D}$ if $\sum_{n}\left|a_{n}-1\right|+\left|b_{n}\right|<\infty$ and, most importantly, identify what $c_{n}$ and $g_{n}$ are, namely,

$$
c_{n}(z)=z^{n} p_{n}\left(z+\frac{1}{z}\right)
$$

where $p_{n}$ are the orthonormal polynomials. Moreover, if $\tilde{J}_{\ell}$ is defined like $J_{\ell}$ (see (6.21) / (6.22) $)$ but with a different cutoff on $a_{j}$, that is,

$$
\begin{aligned}
& \tilde{a}_{j}^{(\ell)}= \begin{cases}a_{j} & j \leq \ell \\
1 & j \geq \ell+1\end{cases} \\
& \tilde{b}_{j}^{(\ell)}= \begin{cases}b_{j} & j \leq \ell \\
0 & j \geq \ell+1\end{cases}
\end{aligned}
$$

then

$$
g_{n}(z, J)=u\left(z, \tilde{J}_{n}\right)
$$

the conventional Jost function for $\tilde{J}_{n}$, that is, $\left(\lim _{m \rightarrow \infty} z^{-m} w_{m}\left(z, \tilde{J}_{n}\right)\right)^{-1}$.

Our goal here is to extend Theorem 5.1 by proving:

Theorem 7.1. The following are equivalent:

(a) For some $\varepsilon \in(0,1), \lim _{n \rightarrow \infty} c_{n}(z)$ exists for $|z|=\varepsilon$ uniformly in such $z$.

(b) For all $z \in \mathbb{D}, \lim _{n \rightarrow \infty} c_{n}(z)$ and $\lim _{n \rightarrow \infty} g_{n}(z)$ exist uniformly on compacts of $\mathbb{D}$, and $\lim g_{n}(z)$ is the Jost function $u(z ; J)$.

(c) Conditions $(\alpha)-(\gamma)$ of Theorem 5.1 hold.

Proof. That (a) $\Rightarrow$ (c) is just (ii) $\Rightarrow$ (iv) in Theorem 5.1. and (b) $\Rightarrow$ (a) is trivial. So we only need (c) $\Rightarrow(\mathrm{b})$. Convergence of $c_{n}$ is just (iv) 
$\Rightarrow$ (i) of Theorem 5.1. so we only need convergence of $g_{n}$. To see this, we use (7.7), (6.17), and (6.27). $\left\|\tilde{J}_{n}-J\right\|_{2} \rightarrow 0$, so

$$
\operatorname{det}_{2}\left(1+\delta \tilde{J}_{n}\left(J_{0}-\left(z+z^{-1}\right)\right)^{-1}\right) \rightarrow \operatorname{det}_{2}\left(1+\delta J\left(J_{0}-\left(z+z^{-1}\right)\right)^{-1}\right)
$$

Clearly, $T\left(z ; \tilde{J}_{n}\right) \rightarrow T(z ; J)$. Thus, $g_{n}$ converges to the Jost function for $J$.

The point of this theorem is that we establish the validity of the GC equations for defining $u$ in the general context of Theorem 5.1. There is a second point - we want to turn this analysis around and directly use the GC equations to prove that, when $(\alpha)-(\gamma)$ hold, $c_{n}(z)$ and $g_{n}(z)$ have limits for $z \in \mathbb{D}$, thereby providing a third proof of the hard part of Theorem 5.1. The key is the following theorem of Coffman [5]:

Theorem $7.2([5])$. Let $J$ be $a d \times d$ diagonal matrix with entries $\lambda_{1}, \ldots, \lambda_{d}$ along the diagonal. Let $A_{n}$ be a sequence of $d \times d$ matrices so

(i)

$$
\sum_{n=1}^{\infty}\left\|A_{n}\right\|^{2}<\infty
$$

(ii) $J$ and $\left\{J+A_{n}\right\}_{n=1}^{\infty}$ are all invertible.

Consider solutions $y_{n} \in \mathbb{C}^{d}$ of

$$
y_{n+1}=\left(J+A_{n}\right) y_{n}
$$

with some initial condition $y_{1}$. Suppose $\lambda_{j}$ is a simple eigenvalue with $\left|\lambda_{j}\right| \neq\left|\lambda_{\ell}\right|$ for $\ell \neq j$. Let

$$
f(n)=\prod_{m=1}^{n-1}\left[\lambda_{j}+\left(A_{m}\right)_{j j}\right]
$$

Then there exists an initial condition $y_{1}$ so that

$$
\lim _{n \rightarrow \infty} \frac{y_{n, j}}{f(n)}
$$

exists and is nonzero, while for $\ell \neq j$,

$$
\lim _{n \rightarrow \infty} \frac{y_{n, \ell}}{f(n)}=0
$$

Remarks. 1. Coffman's result is a discrete analog of continuum (ODE) results of Hartman-Wintner [18]. Related work includes Ford [13], Benzaid-Lutz [1], and Janas-Moszyński [19].

2. Coffman [5] only requires that $\lambda_{j}$ be a simple eigenvalue and allows others can have Jordan blocks. In (7.9), he allows 2 to be replaced by $p \in[1,2]$, but such assumptions imply (7.9)! 
3. A pedagogical presentation of Theorems [7.1 and 7.2 will appear in the second edition of 41. Until that second edition appears, the section will be available on the web at http://www.math.caltech.edu/opuc.html.

Corollary 7.3. Let $J$ be a $d \times d$ matrix with simple eigenvalues $\lambda_{1}=1$, $\lambda_{2}, \ldots, \lambda_{d}$ with $\left|\lambda_{i}\right| \neq\left|\lambda_{j}\right|$ if $i \neq j$ and $\lambda_{2}, \ldots, \lambda_{d} \in \mathbb{D}$. Let $y^{(0)}$ be the eigenvector of $J$ with $J y^{(0)}=y^{(0)}$. Suppose $A_{n}$ obeys (7.9) and

$$
\lim _{N \rightarrow \infty} \sum_{n=1}^{N} A_{n}
$$

exists. Then for any initial condition $y_{1}$, the solution of (17.10) obeys

$$
\lim _{n \rightarrow \infty} y_{n}=c\left(y_{1}\right) y^{(0)}
$$

As a preliminary, note that if $\sum_{n=1}^{N} a_{n}$ has a limit and

$$
\sum_{n=1}^{\infty}\left|a_{n}\right|^{2}<\infty
$$

then $\prod_{j=1}^{N}\left(1+a_{n}\right)$ has a finite limit which is nonzero if all $a_{n} \neq-1$. For $\sum_{n=1}^{\infty} \log \left(1+a_{n}\right)-a_{n}$ is absolutely convergent by (7.15).

Proof. By this remark and Theorem [7.2, there are solutions $y_{n}^{(k)}$ with $y_{n}^{(k)} \lambda_{k}^{-n} \rightarrow$ multiple of eigenvector of $J$ with eigenvalue $\lambda_{k}$ (17.14) follows since $\lambda_{1}=1$ while $\left|\lambda_{k}\right|<1$ for $k \neq 1$.

Remark. By using Perron's theorem, one can show that only $\left|\lambda_{j}\right|<1$ for $j \geq 2$ is needed, not $\left|\lambda_{j}\right| \neq\left|\lambda_{k}\right|$.

Here is the promised third proof of the hard part of Theorem 5.1]

Theorem 7.4. Let conditions $(\alpha)-(\gamma)$ of Theorem 5.1 hold. Let $c_{n}, g_{n}$ be defined by (7.1)-(7.3). Then

$$
\left(\begin{array}{l}
c_{n} \\
g_{n}
\end{array}\right)(z) \rightarrow f(z)\left(\begin{array}{c}
\left(1-z^{2}\right)^{-1} \\
1
\end{array}\right)
$$

Proof. Let $J=\left(\begin{array}{cc}z^{2} & 1 \\ 0 & 1\end{array}\right)$ with $z \in \mathbb{D}$. $J$ has eigenvalues $z^{2} \in \mathbb{D}$ and 1 , and the eigenvector for eigenvalue 1 is $\left(\left(1-z^{2}\right)^{-1} 1\right)^{t}$. Let

$$
A_{n}=\left(\begin{array}{cc}
-b_{n+1} z & 0 \\
\left(1-a_{n+1}^{2}\right) z^{2}-b_{n+1} z & 0
\end{array}\right)
$$

which obeys the hypothesis of Corollary 7.3 by $(\alpha)-(\gamma)$. This corollary plus existence of the limit $\prod_{j=1}^{\infty} a_{j}$ imply (17.16). 
Remark. One can also apply Theorem 7.2 directly to the recursion relation (2.1) to see that there exist solutions $\sim C z^{n}$, that is, Jost solutions.

\section{8. $L^{2}$ Convergence on the Boundary}

Our goal in this section is to prove:

Theorem 8.1. Let $d \rho$ have the form (1.1) and suppose $\left\{a_{n}, b_{n}\right\}_{n=1}^{\infty}$ obey $(\alpha)-(\gamma)$ of Theorem 5.1. Then

$$
\lim _{n \rightarrow \infty} \int_{-2}^{2}\left|p_{n}(x)-(\sin \theta)^{-1} \operatorname{Im}\left(\bar{u}\left(e^{i \theta}\right) e^{i(n+1) \theta}\right)\right|^{2} f(x) d x=0
$$

with $\theta=\arccos \left(\frac{x}{2}\right)$, and

$$
\lim _{n \rightarrow \infty} \int\left|p_{n}(x)\right|^{2} d \rho_{\mathrm{s}}(x)=0
$$

Remark. Unfortunately, there are some errors in the analogous formula in 42, namely, (13.3.15) should have

$$
\frac{\bar{u}(x) e^{i(n+1) \theta}-u(x) e^{-i(n+1) \theta}}{2 i \sin \theta}
$$

where it has

$$
\frac{\bar{u}(x) e^{i(n-1) \theta}-u(x) e^{i(n-1) \theta}}{4 \sin \theta}
$$

and $p_{n}$, not $P_{n}$. As a check, when $b_{n} \equiv 0, a_{n} \equiv 1, p_{n}(2 \cos \theta)=\frac{\sin (n+1) \theta}{\sin \theta}$ and $u \equiv 1$.

This is an analog of what Szegö proved in 46 for OPUC and then translated 47. to exactly this form for OPRL with $\operatorname{supp}(d \rho) \subset[-2,2]$. Peherstorfer-Yudistkii 32 proved precisely this when (1.5) and (1.13) hold. While the underlying core idea behind the proof we use is that of those authors, our technicalities are much more complex.

For all these proofs, the key is to prove what is essentially a weak $L^{2}$ convergence that in the current context is

$$
\lim _{n \rightarrow \infty} \int_{0}^{2 \pi} e^{i n \theta} p_{n}(2 \cos \theta) u\left(e^{i \theta}\right)^{-1}\left(1-e^{2 i \theta}\right) \frac{d \theta}{2 \pi}=1
$$

In Szegö case, $u(z)^{-1}$ is an $H^{2}$-function, so the left side of 8.5 is just $\left.\left[z^{n} p_{n}\left(z+\frac{1}{z}\right) u(z)^{-1}\left(1-z^{2}\right)\right]\right|_{z=0}$, which converges to 1 by the asymptotic result inside the circle. If there are finitely many bound states, $u(z)^{-1}$ has finitely many poles. Using the fact that eigenfunctions go to zero, it is easy to accommodate the poles. For the case that 
Peherstorfer-Yuditskii study, the argument is more subtle but, by cutting off Blaschke products, still involves a contour integral around the whole unit circle.

In contrast, our $u(z)^{-1}$ is so singular at \pm 1 that we do not see how to directly deal with the integral in (8.1). Instead, we will deal with arcs by mapping a sector to the unit disk and relating this to distributional convergence of suitable boundary values of analytic functions. As noted in the introduction, this argument has some elements in common with work of Denisov-Kupin [10] which was done subsequently to our work. In turn, we were all motivated by some arguments of Killip 23.

The technical core of our proof is the following:

Proposition 8.2. Let $(\alpha)-(\gamma)$ of Theorem 5.1 hold. Fix a sector on $\mathbb{D}$,

$$
S=\left\{z|| z \mid<1,0<\theta_{0} \leq \arg z \leq \theta_{1}<\pi\right\}
$$

Then there exist $N$ and $C$ so that for all $n$ and all $z \in S$,

$$
\left|z^{n} p_{n}\left(z+\frac{1}{z}\right)\left(1-z^{2}\right) u(z)^{-1}\right| \leq C(1-|z|)^{-N}
$$

Moreover, $C$ and $N$ are uniformly bounded for $S$ fixed for all $\left\{a_{n}, b_{n}\right\}_{n=1}^{\infty}$ with

$$
\sup _{N}\left(\left|\sum_{n=1}^{N} \log \left(a_{n}\right)\right|+\left|\sum_{n=1}^{N} b_{n}\right|\right)+\sum_{n=1}^{\infty}\left|a_{n}-1\right|^{2}+\left|b_{n}\right|^{2}<K
$$

Remarks. 1. What is critical is the $C$ and $N$ are $n$ independent. $N$ is also $S$ independent, but $C$ is $S$ dependent and diverges as $S$ approaches the real axis, that is, as we approach the singularities at $z= \pm 1$.

2. As noted in the introduction, by using ideas of Denisov-Kupin [10], we can likely prove this with $N=1$; we will have $N=\frac{5}{2}$.

3. We defer the proof until the end of the section.

Definition. If $f(z)$ is a function analytic on $\mathbb{D}$, with

$$
f(z)=\sum_{n=0}^{\infty} \hat{f}(n) z^{n}
$$

and if $f$ obeys

$$
|f(z)| \leq C(1-|z|)^{-N}
$$

SO

$$
|\hat{f}(n)| \leq 4 C(n+1)^{N}
$$


(obtained by writing $\hat{f}(n)$ as a contour integral over a circle of radius $\left.1-(n+1)^{-1}\right)$, we define the distributional boundary values of $f$ by

$$
\int f\left(e^{i \theta}\right) g\left(e^{i \theta}\right) \frac{d \theta}{2 \pi} \equiv \sum_{n=0}^{\infty} \hat{f}(n) \int e^{i n \theta} g\left(e^{i \theta}\right) \frac{d \theta}{2 \pi}
$$

for $C^{\infty}$ functions $g$ on $\partial \mathbb{D}$.

Power bounds like (8.7) are important because of

Proposition 8.3. Let $f_{n}(z)$ be a sequence of functions analytic on $\mathbb{D}$ so that for some fixed $C, N$ and all $n$,

$$
\left|f_{n}(z)\right| \leq C(1-|z|)^{-N}
$$

Suppose $f_{n} \rightarrow f_{\infty}$ uniformly on compacts of $\mathbb{D}$. Then the distributional boundary values converge in (weak) distributional sense.

Proof. Let $f \in C^{\infty}(\partial \mathbb{D})$ and $\hat{g}(-n)$ the integral in (8.12). Write

$$
\sum_{k=0}^{\infty}\left|\hat{f}_{n}(k)-\hat{f}_{\infty}(k)\right||\hat{g}(-k)| \leq 1+2
$$

where $2=\sum_{k=K+1}^{\infty}$ and $1=\sum_{k=1}^{K}$. Then, by (8.11) and (8.13), for $g$ fixed, we can choose $K$ so $\| 2 \mid<\varepsilon$. By the assumed convergence, $\hat{f}_{n}(k) \rightarrow \hat{f}_{\infty}(k)$ for each $k$, so $1 \rightarrow 0$.

Proposition 8.4. For any sector $S$ of the form (8.6), there is an analytic bijection $\varphi: \mathbb{D} \rightarrow S$ and constant $C$ so that

$$
(1-|\varphi(z)|)^{-1} \leq C(1-|z|)^{-1}
$$

Proof. By compactness, we only need to prove this near points where $|\varphi(z)|=1$. Such points are on the part of $\partial \mathbb{D}$ that maps into $\partial S \cap \partial \mathbb{D}$, that is, an arc. At interior points, $\varphi$ is locally linear and (8.14) holds, so we only need to worry about neighborhoods, $N$, of the points that map to corners. In suitable local coordinates, $\zeta$, the corner maps to 0 , $N \cap \mathbb{D}$ maps to $\mathbb{C}_{+} \cap\{\zeta|| \zeta \mid<\varepsilon\}$ and $\varphi$ is transformed to $\varphi(z(\zeta))=\sqrt{\zeta}$ mapping $\mathbb{C}_{+}$to a $90^{\circ}$ corner. In these local coordinates, $1-|\varphi(z)| \sim$ $\operatorname{Im} \sqrt{\zeta}$ and $1-|z| \sim \operatorname{Im} \zeta$, and (8.14) says

$$
\frac{1}{\operatorname{Im} \sqrt{\zeta}}=\frac{2 \operatorname{Re} \sqrt{\zeta}}{\operatorname{Im} \zeta} \leq \frac{C}{\operatorname{Im} \zeta}
$$

which is immediate since $|\zeta|$ is small. Thus, (8.14) holds locally, and so globally. 
Given a sector $S$ of the form (8.6) and $\varphi: \mathbb{D} \rightarrow S$, we can define analytic functions on $\mathbb{D}$,

$$
f_{n}(z)=g_{n}(\varphi(z))
$$

where

$$
g_{n}(z)=z^{n} p_{n}\left(z+\frac{1}{z}\right) u(z)^{-1}\left(1-z^{2}\right)
$$

This allows us to consider boundary values of $f_{n}$, and so $g_{n}$, as distributions. But $g_{n}$, and so $f_{n}$, also has pointwise boundary values, and we want to prove that the distributional boundary value is given by the function. We have

Lemma 8.5. For any sector $S$ of the form (8.6), there is an $\mathbb{H}^{2}$ function $h$ and a function $q$ analytic in a neighborhood of $\mathbb{D} \cup \bar{S}$ so that

$$
u(z)^{-1}=h(z) q(z)
$$

Remark. The point is that $q(z)$ is analytic in $\partial S$, so the boundary values of $u^{-1}$ on $\partial S$ are given by the well-studied theory of boundary values of $\mathbb{H}^{2}$ functions [34, 11.

Proof. We use the representation (5.31). Define

$$
H(\theta)= \begin{cases}\operatorname{Im} M\left(e^{i \theta}\right) & e^{i \theta} \in T \cup \bar{T} \\ |\sin \theta| & e^{i \theta} \notin T \cup \bar{T}\end{cases}
$$

where $T$ is a slightly enlarged $\partial S \cap \partial \mathbb{D}$, but not so enlarged that it includes +1 or -1 . Let

$$
h(z)=\exp \left(\frac{1}{4 \pi} \int \frac{e^{i \theta}+z}{e^{i \theta}-z} \log \left(\frac{H(\theta)}{\sin \theta}\right) d \theta\right)
$$

Since $\frac{H(\theta)}{\sin \theta}>0$ (by (5.32) $)$,

$$
\int \log \left(\frac{H(\theta)}{\sin \theta}\right)>-\infty
$$

and, by (5.32) again,

$$
\int\left(\frac{H(\theta)}{\sin \theta}\right) \frac{d \theta}{2 \pi}<\infty
$$

the function (8.19) is in $\mathbb{H}^{2}$ by the standard approximant argument of Szegő (see [1, Section 2.4]).

Thus, we define $q(z)=u(z)^{-1} h(z)^{-1}$ and need to show that it is analytic in a neighborhood of $\mathbb{D} \cup S$. By (5.31), we can write

$$
q(z)=q_{1}(z) q_{2}(z) q_{3}(z) q_{4}(z)
$$


where

$$
q_{1}(z)=\left(\prod_{j=1}^{\infty} a_{j}\right)^{\alpha(a)} \exp \left(\frac{1}{2} \beta(z) \sum_{j=1}^{\infty} b_{j}\right)
$$

is clearly analytic away from \pm 1 . We have that $q_{2}$ is the inverse of the renormalized Blaschke product is analytic away from $\mathbb{R}$ by Theorem 3.3 . Next,

$$
q_{3}(z)=\exp \left(\frac{1}{4 \pi} \int Q\left(z, e^{i \theta}\right) \log \left(\frac{\operatorname{Im} M}{H(\theta)}\right) d \theta\right)
$$

is analytic since $\log \left(\frac{\operatorname{Im} M}{H(\theta)}\right)$ is supported on $\partial \mathbb{D} \backslash(T \cup \bar{T})$ and $Q\left(z, e^{i \theta}\right)$ has singularities only at $z= \pm 1, \pm e^{i \theta}$. Finally,

$$
q_{4}(z)=\exp \left(\frac{1}{4 \pi} \int\left[Q\left(z, e^{i \theta}\right)-P\left(z, e^{i \theta}\right)\right] \log \left(\frac{H(\theta)}{\sin \theta}\right) d \theta\right)
$$

is analytic since $\log \left(\frac{H(\theta)}{\sin \theta}\right)$ is even, so we can replace $Q-P$ by $Q\left(z, e^{i \theta}\right)-$ $\frac{1}{2} P\left(z, e^{i \theta}\right)-\frac{1}{2} P\left(z, e^{i \theta}\right)$ and this kernel is only singular at $z= \pm 1$.

Given $\theta_{0} \in[0,2 \pi)$, let $R_{\theta_{0}}$ be the region

$$
R_{\theta_{0}}=\left\{z|1>| z \mid>\frac{1}{2}, \arg \left(1-e^{-i \theta_{0}} z\right)<\frac{\pi}{4}\right\}
$$

a region of nontangential approach to $\theta_{0}$. Define the maximal function,

$$
M\left(\theta_{0}\right)=\sup _{z \in R_{\theta_{0}}}\left|u(z)^{-1}\right|
$$

Given Lemma 8.5], standard $\mathbb{H}^{2}$ theory [34, 11, 22] implies that

Proposition 8.6. $u\left(r e^{i \theta}\right)^{-1}$ has boundary values as $r \uparrow 1$ for a.e. $\theta \in$ $(0,2 \pi)$. Indeed, for a.e. $\theta$,

$$
\lim _{\substack{|z| \uparrow 1 \\ z \in R_{\theta_{0}}}} u(z)^{-1}=u\left(e^{i \theta_{0}}\right)^{-1}
$$

Moreover, for every $\eta>0$,

$$
\int_{\eta}^{\pi-\eta} M\left(\theta_{0}\right)^{2} \frac{d \theta}{2 \pi}<\infty
$$

This implies

Proposition 8.7. Let $S$ be a sector of the form (8.6) and $f_{n}$ be given by (8.15) / (8.16). Let $\tilde{S} \subset \partial \mathbb{D}$ be the image of $\partial S \cap \partial \mathbb{D}$ under $\varphi^{-1}$ and let $T_{f_{n}}$ denote the distribution induced by $f_{n}$ on $\partial \mathbb{D}$. Let $t(\theta)$ be a function in $C^{\infty}\left(\tilde{S}^{\text {int }}\right)$. Then

$$
T_{f_{n}}(t)=\int_{\tilde{S}} t(\theta) g_{n}\left(\varphi\left(e^{i \theta}\right)\right) \frac{d \theta}{2 \pi}
$$


where $g_{n}$ is defined by the pointwise boundary value of $u^{-1}$.

Proof. By the definition of (8.12) and the absolute convergence of the sum,

$$
T_{f_{n}}(t)=\lim _{r \uparrow 1} \int_{\tilde{S}} t(\theta) g_{n}\left(\varphi\left(r e^{i \theta}\right)\right) \frac{d \theta}{2 \pi}
$$

By the continuity of $\varphi \mid\left\{r e^{i \eta} \mid \eta \in \operatorname{supp}(t)\right\}$, for all $r$ close enough to $1, \varphi\left(r e^{i \theta}\right) \in R_{\varphi\left(e^{i \theta}\right)}$, so $g_{n}\left(\varphi\left(r e^{i \theta}\right)\right) \rightarrow g_{n}\left(\varphi\left(e^{i \theta}\right)\right)$ by (8.28), and by (8.29) and $\left|t g_{n}\right| \leq 2|t| M \sup _{|t| \leq 1}\left|z^{n} p_{n}\left(z+\frac{1}{z}\right)\right|$, we have domination by a function in $L^{2}$, and so in $L^{1}$. Thus, (8.30) follows from the dominated convergence theorem.

Proposition 8.8. For each $C^{\infty}$ function, let $S$ be a sector of the form (8.6) and $\varphi$ an analytic map of $\mathbb{D}$ to $S$. Let $t$ be a $C^{\infty}$ function with support in $S^{\text {int }}$. Then

$$
\lim _{n \rightarrow \infty} \int_{\tilde{S}} t(\theta) g_{n}\left(\varphi\left(e^{i \theta}\right)\right) \frac{d \theta}{2 \pi}=\int t(\theta) \frac{d \theta}{2 \pi}
$$

Proof. By (8.7) and (8.14), plus Theorem 5.1 (which implies $g_{n}(z) \rightarrow 1$ for $z \in \mathbb{D}$ and so for $z \in S$ ) and Proposition 8.3. $T_{f_{n}} \rightarrow 1$ in distributional sense. This implies (8.32), given Proposition 8.7

These lengthly preliminaries imply the key to $L^{2}$ convergence on the boundary:

Theorem 8.9. Let $u\left(e^{i \theta}\right)^{-1}$ be the boundary values of $u^{-1}$ on $\partial \mathbb{D}$. Let $g_{n}$ be given by (8.16) on $\partial \mathbb{D}$. Then

(2) $g_{n} \rightarrow 1$ in weak- $L^{2}\left(\partial \mathbb{D}, \frac{d \theta}{2 \pi}\right)$.

$$
\int_{0}^{2 \pi}\left|g_{n}\left(e^{i \theta}\right)\right|^{2} \frac{d \theta}{2 \pi} \leq 2
$$

Proof. (1) We begin with some preliminaries concerning the measure $d \mu_{\mathrm{ac}}$ on $\partial \mathbb{D}$ obtained by using $\theta=\arccos \left(\frac{x}{2}\right)$ to move the a.c. part of $d \rho$, that is, $f(x) d x$ to $\partial \mathbb{D}$. Since $e^{i \theta} \rightarrow 2 \cos \theta$ is $2-1$ from $\partial \mathbb{D}$ to $[-2,2]$ (see [42, Section 13.15]),

$$
d \mu_{\mathrm{ac}}(\theta)=|\sin \theta| f(2 \cos \theta) d \theta
$$

By (5.3) and standard theory of Stieltjes transforms,

$$
f(2 \cos \theta)=\frac{\left|\operatorname{Im} M\left(e^{i \theta}\right)\right|}{\pi}
$$

so, by (5.32),

$$
d \mu_{\mathrm{ac}}=\frac{\sin ^{2}(\theta)}{\left|u\left(e^{i \theta}\right)\right|^{2}} \frac{d \theta}{\pi}
$$




$$
=\frac{1}{2} \frac{\left|1-e^{2 i \theta}\right|^{2}}{\left|u\left(e^{i \theta}\right)\right|^{2}} \frac{d \theta}{2 \pi}
$$

Thus,

$$
\begin{aligned}
\int_{0}^{2 \pi}\left|g_{n}\left(e^{i \theta}\right)\right|^{2} \frac{d \theta}{2 \pi} & =2 \int_{0}^{2 \pi}\left|p_{n}(2 \cos \theta)\right|^{2} d \mu_{\mathrm{ac}}(\theta) \\
& =2 \int_{-2}^{2}\left|p_{n}(x)\right|^{2} d \rho_{\mathrm{ac}}(x) \\
& \leq 2 \int_{-2}^{2}\left|p_{n}(x)\right|^{2} d \rho(x)=2
\end{aligned}
$$

proving (8.33).

(2) By (1), the functions $g_{n}$ are uniformly bounded in $L^{2}$, so it suffices to prove that

$$
\int t\left(e^{i \theta}\right) g_{n}\left(e^{i \theta}\right) \frac{d \theta}{2 \pi} \rightarrow \int t\left(e^{i \theta}\right) \frac{d \theta}{2 \pi}
$$

for a total set of $t$ 's. If $t$ is $C^{\infty}$ and supported in some sector $S$ of the form (8.6), (8.38) follows from Proposition 8.8 (there is a Jacobian to go from $d \varphi(\theta)$ to $d \theta$, but it is $C^{\infty}$ on $S^{\text {int }}$ and occurs on both sides of (8.38) ). Since such $t$ 's are total, (2) is proven.

Proof of Theorem 8.1. Define in $L^{2}([-2,2], f(x) d x)$,

$$
\begin{aligned}
& j_{n}^{+}(x)=(2 \sin \theta)^{-1} \overline{u\left(e^{i \theta}\right)} e^{i(n+1) \theta} \\
& j_{n}^{-}(x)=\overline{j_{n}^{+}(x)}
\end{aligned}
$$

where $\theta(x) \in(0, \pi)$ is given by $x=2 \cos (\theta(x))$. By (8.35) and (5.32),

$$
f(x)=\frac{\sin \theta}{\pi\left|u\left(e^{i \theta}\right)\right|^{2}}
$$

Thus, by a change of variables,

$$
\begin{aligned}
\int_{-2}^{2}\left|j_{n}^{+}(x)\right|^{2} f(x) d x & =\int_{-2}^{2} \frac{1}{4} \sin ^{-2} \theta|u|^{2} \frac{\sin \theta}{\pi|u|^{2}} d x \\
& =\frac{1}{2} \int_{0}^{\pi} \frac{d \theta}{\pi}=\frac{1}{2}
\end{aligned}
$$

On the other hand, by the same change of variables,

$$
\begin{aligned}
\left\langle j_{n}^{-}, p_{n}\right\rangle_{L^{2}(f d x)} & =\int_{-2}^{2}(2 \sin \theta)^{-1} \overline{u\left(e^{i \theta}\right)} e^{i n \theta} e^{i \theta} p_{n}(2 \cos \theta) \frac{\sin \theta}{\pi|u|^{2}} d x \\
& =\int_{0}^{\pi} u\left(e^{i \theta}\right)^{-1}\left[\left.\left(1-z^{2}\right) z^{n} p_{n}\left(z+\frac{1}{z}\right)\right|_{z=e^{i \theta}}\right] \frac{-1}{2 i} \frac{d \theta}{\pi}
\end{aligned}
$$




$$
\rightarrow \frac{i}{2}
$$

by Theorem 8.9. (8.43) uses

$$
e^{i \theta} \frac{1}{2 \sin \theta} \sin \theta d(2 \cos \theta)=e^{i \theta} \sin \theta=\frac{-1}{2 i}\left(1-e^{2 i \theta}\right)
$$

Similarly, since $p_{n}$ is real,

$$
\left\langle j_{n}^{+}, p_{n}\right\rangle_{L^{2}(f d x)} \rightarrow-\frac{i}{2}
$$

Finally, by the same change of variables that led to (8.42),

$$
\left\langle j_{n}^{+}, j_{n}^{-}\right\rangle_{L^{2}(f d x)}=\frac{1}{2} \int_{0}^{\pi} e^{-2 i(n+1) \theta} \frac{u\left(e^{i \theta}\right)}{\overline{u\left(e^{i \theta}\right)}} \frac{d \theta}{\pi} \rightarrow 0
$$

since $\frac{u}{\bar{u}} \in L^{2}\left(\partial \mathbb{D}, \frac{d \theta}{2 \pi}\right)$ and $e^{-2 i(n+1) \theta)} \rightarrow 0$ weakly.

Now,

$$
p_{n}(x)-(\sin \theta)^{-1} \operatorname{Im}\left(\bar{u} e^{i(n+1) \theta}\right)=p_{n}(x)-i^{-1}\left[j_{n}^{+}-j_{n}^{-}\right]
$$

so, by (8.42), (8.43), (8.45), and (8.44) (all norms in $L^{2}(f d x)$ ),

$$
\begin{aligned}
0 \leq & \lim \inf \| \text { LHS of }\left(\text { 8.47) } \|^{2}\right. \\
= & \liminf \left(\left\|p_{n}\right\|^{2}+\left\|j_{n}^{+}\right\|^{2}+\left\|j_{n}^{-}\right\|^{2}-2 \operatorname{Re}\left\langle j_{n}^{+}, j_{n}^{-}\right\rangle\right. \\
& \left.\quad+2 \operatorname{Re}\left\langle p_{n}, i j_{n}^{+}\right\rangle-2 \operatorname{Re}\left\langle p_{n}, i j_{n}^{-}\right\rangle\right) \\
& =\liminf \left(\left\|p_{n}\right\|^{2}+\frac{1}{2}+\frac{1}{2}-0-1-1\right) \\
= & \liminf \left\|p_{n}\right\|^{2}-1
\end{aligned}
$$

Thus, since $\left\|p_{n}\right\|_{L^{2}(f d x)}^{2} \leq 1$, we conclude

$$
\lim \left\|p_{n}\right\|_{L^{2}(f d x)}^{2}=1
$$

so $\left\|p_{n}\right\|_{L^{2}\left(d \rho_{\mathrm{s}}\right)} \rightarrow 0$ and, by the above calculation, LHS of (8.47) $\rightarrow$ 0 .

Thus, Theorem 8.1] is reduced to the proof of Proposition 8.2, to which we now turn. As a preliminary, we want to exploit the proof of Lemma 8.5 .

Proposition 8.10. Let $S$ be a sector of the form (8.6) and let $K$ be given. Then there exists $C$ depending only on $S$ and $K$ so that if (8.8) holds, then for $z \in S$,

$$
\left|u(z)^{-1}\right| \leq C(1-|z|)^{-1 / 2}
$$


Proof. As in Lemma 8.5, we construct the factorization (8.17). The proof shows that $\|h\|_{\mathbb{H}^{2}}$ and $\|q\|_{S, \infty} \equiv \sup _{z \in S}|q(z)|$ are bounded by constants $C_{1}$ and $C_{2}$ depending only on $K$ and $S$.

Let $h(z)=\sum_{j=0}^{\infty} h_{j} z^{j}$, then $\|h\|_{\mathbb{H}^{2}}=\left(\sum_{j=0}^{\infty}\left|h_{j}\right|^{2}\right)^{1 / 2}$, so, by the Schwarz inequality,

$$
\begin{aligned}
|h(z)| & \leq\|h\|_{\mathbb{H}^{2}}\left(\sum_{j=0}^{\infty}|z|^{2 j}\right)^{1 / 2} \\
& =\|h\|_{\mathbb{H}^{2}}\left(1-|z|^{2}\right)^{-1 / 2} \\
& \leq C_{1}(1-|z|)^{-1 / 2}
\end{aligned}
$$

so, by (8.17), we have (8.48) with $C=C_{1} C_{2}$.

Our proof will exploit (2.11) where $y_{n}=\tilde{w}_{n}$ and $x_{n}=c_{n-1}$. We are interested in controlling $u^{-1} x_{n}$, which means controlling $u^{-1} y_{n}^{-1}$ and ratios $y_{n} / y_{n-j}$, that is, the functions $u^{-1} \tilde{w}_{n}^{-1}$ and $\tilde{w}_{n} / \tilde{w}_{n-j}$. So we turn first to $u^{-1} \tilde{w}_{n}^{-1}$ and then $\tilde{w}_{n} / \tilde{w}_{n-j}$.

Let $J^{(n)}$ be the Jacobi matrix given after (5.3) and make the $J$ dependence of $w_{n}$ explicit. Then:

Proposition 8.11. Let $\tilde{w}_{n}(z, J)$ be given by (2.2)/(2.3). Then

$$
\tilde{w}_{n}(z, J)^{-1} u(z, J)^{-1}=a_{n} u\left(z, J^{(n)}\right)^{-1}
$$

In particular, if (8.8) holds, then for any $S$ obeying (8.6), there is a $C$ so

$$
\sup _{n}\left|\tilde{w}_{n}(z, J)^{-1} u(z, J)^{-1}\right| \leq C(1-|z|)^{-1 / 2}
$$

Remarks. 1. In order to get (8.48), one does not need a bound on $\sup _{N}\left|\sum_{1}^{N} b_{n}\right|$ but only on $\lim _{N}\left|\sum_{1}^{N} b_{n}\right|$ (and similarly for $\log \left(a_{n}\right)$ ). But to get (8.50), we need control on $\sup _{N} \lim _{M}\left(\sum_{N}^{N+M} b_{n}\right)$ - and that is why we state (8.8) in the form we do.

2. One can also prove this result using the fact that $u w_{n}$ is the unique solution asymptotic to $z^{n}$.

Proof. By (5.7),

$$
\tilde{w}_{n}(z, J)=\left(\frac{M(z)}{z}\right)\left(\frac{a_{1} M_{1}(z)}{z}\right) \ldots\left(\frac{a_{n-1} M_{n-1}(z)}{z}\right)
$$

from which it follows that

$$
\tilde{w}_{n+k}(z, J)=a_{n} \tilde{w}_{n}(z, J) \tilde{w}_{k}\left(z, J^{(n)}\right)
$$

Taking $k$ to infinity using (2.3) and $\tilde{w}_{\infty}=u^{-1}$, we obtain

$$
u(z, J)^{-1}=a_{n} \tilde{w}_{n}(z, J) u\left(z, J^{(n)}\right)^{-1}
$$


which is (8.49).

\section{Proposition 8.12. For any J,}

(i)

$$
\left|\tilde{w}_{n}(z, J)\right| \leq \frac{\pi}{4}|z|^{-n} \varepsilon^{-1}(1-|z|)^{-1}
$$

when $\arg z \in(\varepsilon, \pi-\varepsilon)$ and $0<|z|<1$.

(ii)

$$
\left|\frac{\tilde{w}_{n+k}(z, J)}{\tilde{w}_{n}(z, J)}\right| \leq \frac{\pi}{4} a_{n}|z|^{-k} \varepsilon^{-1}\left(1-|z|^{-1}\right)
$$

when $\arg z \in(\varepsilon, \pi-\varepsilon)$ and $0<|z|<1$.

Proof. (i) By (2.2),

$$
\begin{aligned}
\left|\tilde{w}_{n}(z, J)\right| & \leq \|\left(z+z^{-1}-J\right)^{-1}|||z|^{-n} \\
& \leq\left|\operatorname{Im}\left(z+z^{-1}\right)\right|^{-1}|z|^{-n}
\end{aligned}
$$

since $\sigma(J) \subset \mathbb{R}$ and $J$ is selfadjoint. But if $z=r e^{i \varphi}$ with $0<r<1$,

$$
\left|\operatorname{Im}\left(z+z^{-1}\right)\right|=\left(r^{-1}-r\right)|\sin \varphi|
$$

For $\varphi \in(\varepsilon, \pi-\varepsilon)$,

$$
|\sin \varphi| \geq \frac{2}{\pi} \varepsilon
$$

and

$$
r^{-1}-r=(1-r)\left(r^{-1}+1\right) \geq 2(1-r)
$$

Thus (8.52) holds.

(ii) (8.53) follows immediately from (8.52) and (8.51).

Proof of Proposition 8.2. By (2.11) and the proof of Theorem 2.2 .

$$
\left|c_{n}(z)\right| \leq \sum_{j=0}^{n-1} a_{n-j}^{-1} \frac{\tilde{w}_{n+1}}{\tilde{w}_{n+1-j} \tilde{w}_{n-j}} z^{2 j}+z^{2 n} \frac{\tilde{w}_{n+1}}{\tilde{w}_{1}}
$$

Define

$$
A=\sup _{n}\left(\left|a_{n}\right|,\left|a_{n}\right|^{-1}\right)<\infty
$$

since $a_{n} \rightarrow 1$. Then, by (8.57),

$$
\begin{aligned}
\sup _{n}\left|c_{n}(z) u(z)^{-1}\right| \leq A(1 & -|z|)^{-1} \sup _{n, j}\left|\frac{\tilde{w}_{n+1} z^{j}}{\tilde{w}_{n+1-j}}\right| \sup _{n, j} \frac{1}{\left|\tilde{w}_{n-j} u\right|} \\
& +\sup _{n}\left|\frac{\tilde{w}_{n+1} z^{n}}{\tilde{w}_{1}}\right||u|^{-1}
\end{aligned}
$$


By (8.53), with $\varepsilon$ chosen so $S \subset\{z \mid \arg z \in(\varepsilon, \pi-\varepsilon)\}$, we have

$$
\sup _{n, j}\left|\frac{\tilde{w}_{n+1} z^{j}}{w_{n+i-j}}\right| \leq \frac{\pi A}{4} \varepsilon^{-1}(1-|z|)^{-1}
$$

By (8.50),

$$
\sup _{n, j}\left|\frac{1}{\tilde{w}_{n-j} u}\right| \leq C(1-|z|)^{-1 / 2}
$$

Thus, (8.58) implies that

$$
\left|z^{n} p_{n}\left(z+\frac{1}{z}\right)\left(1-z^{2}\right) u(z)^{-1}\right| \leq C(1-|z|)^{-5 / 2}
$$

where $C$ depends on $S$ and the constant $K$ in (8.8).

This completes the proof of Theorem 8.1. We note that what is missing is proving that wave operators exist. One can use (8.1) to prove that for a.e. $\theta$, the transfer matrix is bounded along a subsequence and so obtain an alternate proof of the Deift-Killip theorem [9] when $(\beta),(\gamma)$ hold. Of course, we use the Killip-Simon sum rule which also implies that theorem. Still it is interesting that one has plane waves like solutions in $L^{2}$ sense in $[-2,2]$. The deeper and interesting open question is pointwise convergence for a.e. $\theta$.

\section{Bound States}

One knows that with regard to Szegö asymptotics, sometimes simplelooking assumptions are really quite restrictive: for instance (see, e.g., 42. Chapter 13]), if $\operatorname{supp}(d \mu) \subset[-2,2]$, then $(\beta)$ of Theorem 5.1 implies all of $(\alpha)-(\gamma)$ and all the other hypotheses of that theorem. Also (see [44]), if $(\beta)$ holds and $f$ is given by (1.1), then

$$
\sum_{j=1, \pm}^{N_{ \pm}}\left(\left|E_{j}^{ \pm}\right|-2\right)^{1 / 2}<\infty
$$

if and only if

$$
\int_{-2}^{2}(\log f)\left(4-x^{2}\right)^{-1 / 2} d x>-\infty
$$

Here we want to show that $(\beta),(\gamma)$ alone do not imply spectral restrictions. In particular, we want to show that for each $q<\frac{3}{2}$, there is a Jacobi matrix obeying $(\alpha)-(\gamma)$ where

$$
\sum_{j=1, \pm}^{N_{ \pm}}\left(\left|E_{j}^{ \pm}\right|-2\right)^{q}=\infty
$$


Of course, by [24], $(\alpha)$ implies

$$
\sum_{j=1, \pm}^{N_{ \pm}}\left(\left|E_{j}^{ \pm}\right|-2\right)^{3 / 2}<\infty
$$

Our construction will have $a_{n} \equiv 1$ and $b_{n}$ nonzero in blocks. In 42 , Section 13.9], examples with $b_{n}$ nonzero in a sequence of isolated points are constructed where $(\alpha)-(\gamma)$ hold and (9.3) holds for $p$ arbitrarily close to 1 . So this section improves that result. Our construction is closely related to that in Theorem 5.12 of [6].

Pick $\alpha$ in $\left(\frac{1}{2}, 1\right)$ and $p$ so that

$$
\frac{\alpha}{1-\alpha}>p>\frac{\alpha}{2-\alpha}
$$

We will eventually take $\alpha$ to $\frac{1}{2}$ and $p-\frac{\alpha}{2-\alpha} \rightarrow 0$. Pick $M_{0}$ and $C_{1}$ so for $m \geq M_{0}$, the distances between the blocks $B_{m} \equiv\left[m^{p+1}-C_{1} m^{p}, m^{p+1}+\right.$ $\left.C_{1} m^{p}\right]$ for $m=M_{0}, M_{0}+1, \ldots$ are each at least 2 . This is easy to do if one fixes $C_{1}<\frac{1}{2}(p+1)$. We should use $\left[C_{1} m^{p}\right]$, but for notational simplicity, we will pretend that $C_{1} m^{p}$ is an integer. We pick $b_{n}$ by

$$
b_{n}= \begin{cases}n^{-\alpha} & n \in B_{2 k}, 2 k \geq M_{0} \\ -n^{-\alpha} & n \in B_{2 k+1}, 2 k+1 \geq M_{0} \\ 0 & \text { otherwise }\end{cases}
$$

Lemma 9.1. $a_{n} \equiv 1$ and $b_{n}$ in (9.6) obey $(\alpha)-(\gamma)$ of Theorem 5.1.

Proof. Since $\left|b_{n}\right| \leq n^{-\alpha}$ and $\alpha>\frac{1}{2}$, condition $(\alpha)$ holds and $(\beta)$ is trivial. So we only need to check $(\gamma)$. Since $\frac{d}{d n} n^{-\alpha}=-\alpha n^{-\alpha-1}$, if $n \in B_{m}$, then

$$
\begin{aligned}
\left|n^{-\alpha}-m^{-(p+1) \alpha}\right| & \leq C m^{-(p+1)(\alpha+1)} m^{p} \\
& =C m^{-[(p+1) \alpha+1]}
\end{aligned}
$$

Thus,

$$
\left|\sum_{n \in B_{m}}\right| b_{n}\left|-2 C_{1} m^{-\alpha(p+1)+p}\right| \leq C_{2} m^{-1} m^{-\alpha(p+1)+p}
$$

We claim

$$
\alpha(p+1)>p
$$

which implies, first, that the estimate on the right of (9.7) is absolutely summable and, second, that $\sum_{m}(-1)^{m} m^{-\alpha(p+1)+p}$ is conditionally summable, proving $(\gamma)$.

To prove (9.8), note that it is equivalent to $\alpha>p(1-\alpha)$ or $p<\frac{\alpha}{1-\alpha}$, which is true by (9.5). 
For $m$ even, we will pick $\varphi_{m}$ to be the trial vector supported in $B_{m}$, which is 1 at the center of $B_{m}$ (i.e., at $n=m^{p+1}$ ), 0 at the end points, and constant slope in between. For $m$ odd, we do the same construction and then multiply by $(-1)^{n}$.

Consider $m$ even first. Since $a_{n} \equiv 1$,

$$
\begin{aligned}
\left\langle\varphi_{m},\left(J_{0}-2\right) \varphi_{m}\right\rangle & =-\sum_{n}\left|\varphi_{m}(n+1)-\varphi_{m}(n)\right|^{2} \\
& \geq-m^{p}\left[\frac{C}{m^{p}}\right]^{2} \\
& =-C^{2} m^{-p}
\end{aligned}
$$

since the slope $\sim m^{-p}$ and there are $O\left(m^{p}\right)$ nonzero terms in the sum (9.9). On the other hand, since $b_{n}>C_{3} m^{-\alpha(p+1)}$ on $B_{m}$ and, on average, $\left|\varphi_{m}\right|^{2} \geq \frac{1}{4}$ on $B_{m}$

$$
\left\langle\varphi_{m}, b \varphi_{m}\right\rangle \geq C_{3} m^{p} m^{-\alpha(p+1)}
$$

It is easy to see that $p>\frac{\alpha}{2-\alpha}$ is equivalent to $2 p>\alpha(p+1)$, so for $m \geq M_{1}$ for some $M_{1}$,

$$
\left\langle\varphi_{m}\left(J_{0}+b-2\right) \varphi_{m}\right\rangle \geq \frac{1}{2} C_{3} m^{p} m^{-\alpha(p+1)}
$$

Since $\left\|\varphi_{m}\right\|^{2} \leq C_{4} m^{p}$, we see that

$$
\frac{\left\langle\varphi_{m},\left(J_{0}+b-2\right) \varphi_{m}\right\rangle}{\left\|\varphi_{m}\right\|^{2}} \geq C_{4} m^{-\alpha(p+1)}
$$

for $m$ even. Similarly, for $m$ odd,

$$
\frac{\left\langle\varphi_{m},\left(J_{0}+2+b\right) \varphi_{m}\right\rangle}{\left\|\varphi_{m}\right\|^{2}} \leq-C_{4} m^{-\alpha(p+1)}
$$

Since $\left\langle\varphi_{m}, \varphi_{k}\right\rangle=0=\left\langle\varphi_{m},\left(J_{0}+b\right) \varphi_{k}\right\rangle$ for $m \neq k$, a variational argument proves for $m$ large,

$$
|| E_{m}^{ \pm}|-2| \geq \frac{1}{2} C_{4} m^{-\alpha(p+1)}
$$

Thus, (9.3) holds if $q \alpha(p+1)<1$. Taking $\alpha \downarrow \frac{1}{2}, p \downarrow \frac{1}{3}$, we see $q \uparrow\left(\left(\frac{1}{2}\right)\left(\frac{4}{3}\right)\right)^{-1}=\frac{3}{2}$. Thus,

Theorem 9.2. For any $q<\frac{3}{2}$, there is a set of Jacobi parameters for which $(\alpha)-(\gamma)$ of Theorem 5.1 hold, but for which (9.3) also holds. 


\section{A Remark on Schrödinger Operators}

In this section, we want to show how the ideas of Section 6 provide a simple proof of

Theorem 10.1. Suppose $V \in L^{2}(0, \infty)$ and

$$
\lim _{x \rightarrow \infty} \int_{0}^{x} V(y) d y
$$

exists. Then for any $\kappa$ with $\kappa>0$, there is a solution of

$$
-u^{\prime \prime}+V u=-\kappa^{2} u
$$

so that

$$
\lim _{x \rightarrow \infty} e^{\kappa x} u(x)=1
$$

This result is not new. It was proven by Hartman-Winter [18] using sophisticated ODE asymptotic methods. Even with the simplification of Harris-Lutz [17, the proof is involved (see Eastham [12] for a particularly clear discussion of this proof). Here, as in Section 6, we will use renormalized determinants to construct $u$. The same argument shows that if (10.1) does not have a finite limit, then there is a solution so $u(x) / \exp [f(x)] \rightarrow 1$, where

$$
f(x)=-\kappa x+\frac{1}{2 \kappa} \int_{0}^{x} V(y) d y
$$

also a result of Hartman-Wintner [18].

In the argument below, we will use unfactorized kernels (i.e., $V G_{0}$ ) rather than factorized kernels (i.e., $V^{1 / 2} G_{0}|V|^{1 / 2}$ ). By using factorized kernels, one should be able to extend this theorem to the case where $V \in L^{2}$ is replaced by $\sum_{n}\left(\int_{n}^{n+1}|V(x)| d x\right)^{2}<\infty$.

The starting point is a formula of Jost-Pais 21 for the Jost function extended to get the Jost solutions.

Proposition 10.2. Let $G_{0}\left(-\kappa^{2}\right)$ be the operator $\left(H_{0}+\kappa^{2}\right)^{-1}$ where $H_{0}$ is $-\frac{d^{2}}{d x^{2}}$ with $u(0)$ boundary conditions, so $G_{0}$ has integral kernel

$$
G_{0}\left(x, y ;-\kappa^{2}\right)=(2 \kappa)^{-1}\left[e^{-\kappa|x-y|}-e^{-\kappa(x+y)}\right]
$$

For any $V \in L^{2}$ of compact support and any $x_{0}>0$, let $K\left(x_{0} ; \kappa\right)$ be the operator with integral kernel

$$
K\left(x, y ; x_{0} ; \kappa\right)=V\left(x+x_{0}\right) G_{0}\left(x, y ;-\kappa^{2}\right)
$$

Then $K$ is trace class and

$$
u\left(x_{0}\right) \equiv e^{-\kappa x_{0}} \operatorname{det}\left(1+K\left(x_{0} ; \kappa\right)\right)
$$


obeys (10.2), and for $x$ large,

$$
u(x)=e^{-\kappa x}
$$

Remark. (10.8) is trivial since $V$ has compact support, which means $K \equiv 0$ for $x_{0}$ large.

Proof. This is essentially Proposition 2.9 of [40]. That paper uses a factorized kernel, but by the Birman-Solomyak theorem (see [38, Chapter 4], $K$ is trace class, and so the determinants are equal. As noted, (10.8) is immediate.

Proposition 10.3. If $V \in L^{2}$ and (10.1) holds, then $K\left(x_{0} ; \kappa\right)$ is Hilbert-Schmidt and

$$
u\left(x_{0}\right)=e^{-\kappa x_{0}} \operatorname{det}_{2}\left(1+K\left(x_{0} ; \kappa\right)\right) \exp \left((2 \kappa)^{-1} \int_{x_{0}}^{\infty} V(y)\left[1-e^{-2 \kappa y}\right] d y\right)
$$

Moreover, $u$ obeys (10.2).

Proof. If $V$ has compact support, (10.9) is just (10.7) since $\operatorname{Tr}\left(K\left(x_{0} ; \kappa\right)\right)=\int_{x_{0}}^{\infty} V(y)(2 \kappa)^{-1}\left[1-e^{-2 \kappa y}\right] d y$ and we have 6.11). Given general $V$, let $V_{L}(x)$ be given by

$$
V_{L}(x)= \begin{cases}V(x) & x \leq L \\ 0 & x>L\end{cases}
$$

and $u_{L}$ given by (10.1). Since $V \in L^{2}, K_{L}\left(x_{0}, \kappa\right) \rightarrow K\left(x_{0} ; \kappa\right)$ in HilbertSchmidt norm, so det ${ }_{2}$ converges. By (10.1), the exponentials converge. Thus, $u_{L} \rightarrow u$. This means $u$ is a distributional solution of (10.2) and so, by elliptic regularity, a solution $L^{2}$ at infinity.

Proof of Theorem 10.1. $K\left(x_{0} ; \kappa\right) \rightarrow 0$ in Hilbert-Schmidt norm as $x_{0} \rightarrow \infty$, so $\operatorname{det}_{2}\left(1+K\left(x_{0} ; \kappa\right)\right) \rightarrow 1$. The integral goes to 0 as $x_{0} \rightarrow \infty$. Thus, $u\left(x_{0}\right) e^{\kappa x_{0}} \rightarrow 1$.

The point here is that it is natural to try to construct $u$ as a limit of $u_{L}$ 's, and then prove asymptotics of $u$. The fact that we have an explicit formula in terms of renormalized determinants allows us to control both the limit as $L \rightarrow \infty$ and then as $x \rightarrow \infty$.

\section{REFERENCES}

[1] Z. Benzaid and D.A. Lutz, Asymptotic representation of solutions of perturbed systems of linear difference equations, Stud. Appl. Math. 77 (1987), 195-221.

[2] T. Carleman, Zur Theorie der linearen Integralgleichungen, Math. Z. 9 (1921), $196-217$. 
[3] K.M. Case, Orthogonal polynomials from the viewpoint of scattering theory, J. Math. Phys. 15 (1974), 2166-2174.

[4] K.M. Case, Orthogonal polynomials, II J. Math. Phys. 16 (1975), 1435-1440.

[5] C.V. Coffman, Asymptotic behavior of solutions of ordinary difference equations, Trans. Amer. Math. Soc. 110 (1964), 22-51.

[6] D. Damanik, D. Hundertmark, R. Killip, and B. Simon, Variational estimates for discrete Schrödinger operators with potentials of indefinite sign, Comm. Math. Phys. 238 (2003), 545-562.

[7] D. Damanik, R. Killip, and B. Simon, Necessary and sufficient conditions in the spectral theory of Jacobi matrices and Schrödinger operators, Int. Math. Res. Not. (2004), no. 22, 1087-1097.

[8] D. Damanik and B. Simon, Jost functions and Jost solutions for Jacobi matrices, II. Decay and Analyticity, preprint.

[9] P.A. Deift and R. Killip, On the absolutely continuous spectrum of onedimensional Schrödinger operators with square summable potentials, Comm. Math. Phys. 203 (1999), 341-347.

[10] S. Denisov and S. Kupin, Asymptotics of the orthogonal polynomials for the Szegö class with a polynomial weight, preprint.

[11] P.L. Duren, Theory of $H^{p}$ Spaces, Pure and Applied Math., 38, Academic Press, New York-London, 1970.

[12] M.S.P. Eastham, The Asymptotic Solution of Linear Differential Systems. Applications of the Levinson Theorem, London Mathematical Society Monographs, New Series, 4. The Clarendon Press, Oxford University Press, New York, 1989.

[13] W. Ford, On the integration of the homogeneous linear difference equation of second order, Trans. Amer. Math. Soc. 10 (1909), 319-336.

[14] J.S. Geronimo and K.M. Case, Scattering theory and polynomials orthogonal on the real line, Trans. Amer. Math. Soc. 258 (1980), 467-494.

[15] I.C. Gohberg and M.G. Krein, Introduction to the Theory of Linear Nonselfadjoint Operators, Transl. Math. Monographs, 18, American Mathematical Society, Providence, R.I., 1969.

[16] A.A. Gončar, On convergence of Padé approximants for some classes of meromorphic functions, Math. USSR Sb. 26 (1975), 555-575.

[17] W.A. Harris and D.A. Lutz, On the asymptotic integration of linear differential systems, J. Math. Anal. Appl. 48 (1974), 1-16.

[18] P. Hartman and A. Wintner, Asymptotic integrations of linear differential equations, Amer. J. Math. 77 (1955), 45-86; errata, 404.

[19] J. Janas and M. Moszyński, Spectral properties of Jacobi matrices by asymptotic analysis, J. Approx. Theory 120 (2003), 309-336.

[20] R. Jost, Über die falschen Nullstellen der Eigenwerte der S-Matrix, Helvetica Phys. Acta 20 (1947), 256-266.

[21] R. Jost and A. Pais, On the scattering of a particle by a static potential, Phys. Rev. 82 (1951), 840-851.

[22] Y. Katznelson, An Introduction to Harmonic Analysis, 2nd corrected edition, Dover Publications, New York, 1976.

[23] R. Killip, Perturbations of one-dimensional Schrödinger operators preserving the absolutely continuous spectrum, Int. Math. Res. Not. (2002), no. 38, 20292061. 
[24] R. Killip and B. Simon, Sum rules for Jacobi matrices and their applications to spectral theory, Ann. of Math. (2) 158 (2003), 253-321.

[25] S. Kupin, On sum rules of special form for Jacobi matrices, C. R. Math. Acad. Sci. Paris 336 (2003), 611-614.

[26] S. Kupin, On a spectral property of Jacobi matrices, Proc. Amer. Math. Soc. 132 (2004), 1377-1383.

[27] A. Laptev, S. Naboko, and O. Safronov, On new relations between spectral properties of Jacobi matrices and their coefficients, Comm. Math. Phys. 241 (2003), 91-110.

[28] N. Levinson, The asymptotic nature of solutions of linear systems of differential equations, Duke Math. J. 15 (1948), 111-126.

[29] F. Nazarov, F. Peherstorfer, A. Volberg, and P. Yuditskii, On generalized sum rules for Jacobi matrices, Int. Math. Res. Not. (2005), no. 3, 155-186.

[30] P. Nevai, Orthogonal polynomials, Mem. Amer. Math. Soc. 18 (1979), no. 213, 185 pp.

[31] E.M. Nikishin, Discrete Sturm-Liouville operators and some problems of function theory, J. Sov. Math. 35 (1986), 2679-2744.

[32] F. Peherstorfer and P. Yuditskii, Asymptotics of orthonormal polynomials in the presence of a denumerable set of mass points, Proc. Amer. Math. Soc. 129 (2001), 3213-3220.

[33] M. Reed and B. Simon, Methods of Modern Mathematical Physics, II. Fourier Analysis, Self-Adjointness, Academic Press, New York, 1975.

[34] W. Rudin, Real and Complex Analysis, 3rd edition, McGraw-Hill, New York, 1987.

[35] E. Seiler, Schwinger functions for the Yukawa model in two dimensions with space-time cutoff, Comm. Math. Phys. 42 (1975), 163-182.

[36] E. Seiler and B. Simon, On finite mass renormalizations in the twodimensional Yukawa model, J. Math. Phys. 16 (1975), 2289-2293.

[37] J.A. Shohat, Théorie Générale des Polinomes Orthogonaux de Tchebichef, Mémorial des Sciences Mathématiques, 66, pp. 1-69, Paris, 1934.

[38] B. Simon, Trace Ideals and Their Applications, Cambridge University Press, Cambridge, 1979.

[39] B. Simon, The classical moment problem as a self-adjoint finite difference operator, Adv. in Math. 137 (1998), 82-203.

[40] B. Simon, Resonances in one dimension and Fredholm determinants, J. Funct. Anal. 178 (2000), 396-420.

[41] B. Simon, Orthogonal Polynomials on the Unit Circle, Part 1: Classical Theory, AMS Colloquium Series, American Mathematical Society, Providence, RI, 2005.

[42] B. Simon, Orthogonal Polynomials on the Unit Circle, Part 2: Spectral Theory, AMS Colloquium Series, American Mathematical Society, Providence, RI, 2005.

[43] B. Simon, A canonical factorization for meromorphic Herglotz functions on the unit disk and sum rules for Jacobi matrices, J. Funct. Anal. 214 (2004), 396-409.

[44] B. Simon and A. Zlatoš, Sum rules and the Szegö condition for orthogonal polynomials on the real line, Comm. Math. Phys. 242 (2003), 393-423. 
[45] G. Szegö, Ein Grenzwertsatz über die Toeplitzschen Determinanten einer reellen positiven Funktion, Math. Ann. 76 (1915), 490-503.

[46] G. Szegö, Beiträge zur Theorie der Toeplitzschen Formen, I, II , Math. Z. 6 (1920), 167-202; ibid. 9 (1921), 167-190.

[47] G. Szegö, Über die Nullstellen von Polynomen, die in einem Kreise gleichmäßig konvergieren, Sitzungsber. Berlin Math. Ges. 21 (1922), 59-64.

[48] G. Szegö, Über den asymptotischen Ausdruck von Polynomen, die durch eine Orthogonalitätseigenschaft definiert sind, Math. Ann. 86 (1922), 114-139.

[49] S. Verblunsky, On positive harmonic functions (second paper), Proc. London Math. Soc. (2) 40 (1936), 290-320. 